\title{
Inverse amplitude method in chiral perturbation theory
}

\author{
A. Dobado* \\ Departamento de Física Teórica, Universidad Complutense, 28040 Madrid, Spain \\ J. R. Peláez ${ }^{\dagger}$ \\ Theoretical Physics Group, Lawrence Berkeley Laboratory, University of California, Berkeley, California 94720
}

(Received 12 November 1996)

\begin{abstract}
Based on a dispersive approach, we apply the inverse amplitude method to unitarize one-loop SU(2) and $\mathrm{SU}(3)$ chiral perturbation theory. Numerically, we find that this unitarization technique yields the correct complex analytic structure in terms of cuts and poles. Indeed, using the chiral parameter estimates obtained from low-energy experiments we obtain the poles associated with the $\rho(770)$ and $K^{*}(982)$ resonances. Just by fixing their actual masses we obtain a parametrization of the $\pi \pi$ and $\pi K$ phase shifts in eight different channels. With this fit we have then calculated several low-energy phenomenological parameters estimating their errors. Among others, we have obtained the chiral parameters and scattering lengths, which can be relevant for future experiments. [S0556-2821(97)00217-8]
\end{abstract}

PACS number(s): 14.40.Aq, 11.80.Et, 13.75.Lb, 14.40.Cs

\section{INTRODUCTION}

Even though QCD yields a remarkably good description of strong interactions, low-energy hadron physics has to be modeled phenomenologically. This is due to the fact that the usual perturbative approach in the coupling constant cannot be applied to QCD below energies of the order of $1 \mathrm{GeV}$. Most of the phenomenological results were based on partial conservation of the axial vector current and current algebra. However, in 1979, Weinberg [1] showed how to reobtain many of these predictions by means of an effective Lagrangian.

The fields in that Lagrangian are the light mesons (pions, kaons, and $\eta$ 's) which are understood as the Goldstone bosons (GB's) arising from the spontaneous breaking of chiral symmetry. The Lagrangian is built as an expansion in derivatives that respects the symmetry-breaking pattern of QCD. Indeed, the first term in the expansion is fixed by the symmetry requirements and accounts for the current algebra results. The next terms in the expansion produce further corrections, which depend on several phenomenological parameters but are always consistent with the QCD symmetry constraints. These techniques were later developed to one loop in a set of papers by Gasser and Leutwyler [2,3]. They showed how to obtain amplitudes involving light mesons, as functions of their momenta, their masses, and those few phenomenological parameters.

By fitting these parameters from a few low-energy experiments it is then possible to obtain successful predictions for other processes. The whole approach is known as chiral perturbation theory (ChPT).

Very recently, some partial higher order calculations [4] as well as a complete two loop calculation of $\pi \pi$ scattering

\footnotetext{
*Electronic address: dobado@eucmax.sim.ucm.es

${ }^{\dagger}$ On leave of absence from Departamento de Física Teórica, Universidad Complutense, 28040 Madrid, Spain. Electronic address: pelaez@theorm.lbl.gov, pelaez@vxcern.cern.ch
}

[5] have appeared in the literature which will be needed in order to analyze new data to come from DAФNE and Brookhaven. For a general review of the available experimental data on pion physics and future prospects, we refer the reader to [6].

Nevertheless, there are some intrinsic limitations when applying ChPT, namely, the fact that the amplitudes calculated within the chiral approach are only unitary in the perturbative sense, that is, up to the next order in the external momenta. Such a breakdown of unitarity is most severe at high energies, where the external momenta are no longer a good expansion parameter, although it can also occur at moderate energies [7]. As a result, it is not possible to reproduce resonant states, which are one of the most characteristic features of the strongly interacting regime. Many different methods have been proposed in order to improve this behavior and thus to extend the applicability of ChPT to higher energies; among them, the use of Padé approximants [8], the explicit introduction of resonances $[9,10]$, the $K$ matrix [11], and the large $N$ limit [12] ( $N$ being the number of GB's) or the inverse amplitude method (IAM) [7,8,13,14].

This work is devoted precisely to the last method, which can be justified within a dispersive approach and can easily reproduce the two lightest resonances: the $\rho(770)$ in $\pi \pi$ scattering [8] and the $K^{*}(892)$ in $\pi K$ scattering [13]. But not only that, the IAM also improves considerably the fit to data even in nonresonant channels, almost up to the first two particle inelastic thresholds. (The many particle inelastic thresholds can be neglected since they are suppressed by phase space factors.) This fit provides a remarkably good parametrization that can be used for other processes. Indeed, in a previous work [15], the authors showed how it can be used together with a simple unitarization prescription to obtain successful results on $\gamma \gamma \rightarrow \pi^{0} \pi^{0}$ up to $700 \mathrm{MeV}$.

Of course, it is also possible to obtain very good parametrizations $[9,10]$ of $\pi \pi$ or $\pi K$ elastic scattering by including all resonant states explicitly. However, our aim choosing the IAM is to reproduce these phenomena just with the few phenomenological parameters present in the ChPT Lagrang- 
TABLE I. Lightest resonances with $\pi \pi$ or $\pi K$ dominant decay modes. Data taken from [16].

\begin{tabular}{lcccc}
\hline \hline Name & $I, J$ & Mass & Width & Dominant decays \\
\hline$\rho(770)$ & 1,1 & $768.8 \pm 1.0$ & $150.3 \pm 1.0$ & $\pi \pi, 100 \%$ \\
$f_{0}(980)$ & 0,0 & $980 \pm 10$ & $40-400$ & $\pi \pi,(78.1 \pm 2.4) \%$ \\
$f_{2}(1270)$ & 0,2 & $1275 \pm 5$ & $185 \pm 20$ & $\pi \bar{K},(21.9 \pm 2.4) \%$ \\
$K^{*}(892)^{ \pm}$ & $1 / 2,1$ & $891.59 \pm 0.24$ & $49.8 \pm 0.8$ & $\pi \pi,(84.7 \pm 2.6) \%$ \\
$K^{*}(892)^{0}$ & $1 / 2,1$ & $896.10 \pm 0.28$ & $50.5 \pm 0.6$ & $\pi K, \simeq 100 \%$ \\
\hline \hline
\end{tabular}

ian. In this way, even though their masses and widths will not be obtained with great accuracy, resonances can be regarded as real predictions. That is one of the relevant features of the IAM since other very popular unitarization methods are not able to reproduce resonances unless they are explicitly introduced in the calculation. That is, for instance, the case with the $K$ matrix.

The purpose of this work is, first, to study how high in energies the IAM yields good results and what are its limitations. We would also like to know whether it is possible to reproduce further resonance states. It is clear that the best candidates are the lightest resonances whose dominant decay modes are $\pi \pi$ or $\pi K$. We have listed them in Table I. In case these resonances were not accommodated after our unitarization, it would be interesting to understand why. Second, once we have a good fit to these resonances, we want to make a complete numerical analysis of several low-energy quantities of interest, such as the chiral parameters or the scattering lengths, including estimations for their errors. As we will see below, we expect that the IAM somehow will include effects that cannot be obtained from the pure $p^{2}$ expansion.

Finally, we would like to comment on another motivation of the present work, which at first may not seem very related to the main topic. The philosophy of the chiral approach has also reached the description of the strongly interacting symmetry-breaking sector (SISBS) of the standard model [17]. The scalar sector of such a model displays the same symmetry-breaking pattern as two flavor massless QCD. Hence, it is possible to build an effective Lagrangian, much as it is done for ChPT [18]. Although the electroweak GB's are not physical, using this Lagrangian it is possible to obtain predictions for the scattering of longitudinal gauge bosons [19] at future colliders, such as the CERN Large Hadron Collider. Indeed, there are already experimental proposals to measure the electroweak chiral parameters at the Compact Muon Solenoid (CMS) [20]. Most of the works on the SISBS make use of the equivalence theorem [17], which allows us to read the observable amplitudes, in terms of longitudinal gauge bosons, directly from those with GB's. This theorem has been recently proved in the chiral Lagrangian formalism [21] and seems to be severely constrained by the lack of unitarity. At this point the unitarization procedures come into play and it is crucial to know whether they are reliable, since what we are now looking for are real predictions and not elaborated fits to still unavailable data.

In Sec. II we review some basic aspects of exact and perturbative unitarity and define the partial waves in elastic scattering. Section III introduces the IAM, first with a deri- vation from dispersion theory and then by studying the constraints to its applicability. Sections IV and V are organized in the same way, although they refer to $S U(2)$ and $S U(3)$ ChPT, respectively: First we apply the IAM to ChPT with the chiral parameters obtained from low-energy experiments in order to study the IAM predictive power. Next, we present an IAM fit to the data. For the best SU(3) fit we present the unitarized results for the scattering lengths and some other phenomenological parameters. Then, in Sec. VI, we study the analytic structure on the complex plane of the IAM amplitudes. In Sec. VII we present the conclusions. There is also an Appendix where we give the elastic scattering formulas used in this work, as well as a discussion on perturbative unitarity.

\section{PARTIAL WAVES, PHASE SHIFTS, AND UNITARITY}

When dealing with strong interactions, it is usual to project the amplitudes in partial waves with definite angular momentum $J$ and isospin $I$ as

$$
t_{I J}(s)=\frac{1}{32 K \pi} \int_{-1}^{1} d(\cos \theta) P_{J}(\cos \theta) T_{I}(s, t)
$$

where $K=2$ or 1 depending on whether the particles in the process are identical or not. The acceptable isospin values also depend on the process, namely, $I=0,1,2$ for $\pi \pi$ elastic scattering and $I=1 / 2,3 / 2$ for $\pi K$. For both reactions the definite isospin amplitudes $T_{I}$ are obtained from a single function. In the first case,

$$
\begin{gathered}
T_{0}(s, t, u)=3 A(s, t, u)+A(t, s, u)+A(u, t, s), \\
T_{1}(s, t, u)=A(t, s, u)-A(u, t, s), \\
T_{2}(s, t, u)=A(t, s, u)+A(u, t, s),
\end{gathered}
$$

whereas for $\pi K$ scattering, we can write

$$
T_{1 / 2}(s, t, u)=\frac{3}{2} T_{3 / 2}(u, t, s)-\frac{1}{2} T_{3 / 2}(s, t, u) .
$$

In order to deal with both processes on the same footing, we will label the particles in the reaction as $\alpha$ and $\beta$. Thus, the Mandelstam variables will satisfy $s+t+u=2\left(M_{\alpha}^{2}\right.$ $\left.+M_{\beta}^{2}\right)$ and the threshold will be at $s_{t h}=\left(M_{\alpha}+M_{\beta}\right)^{2}$. As it is well known, whenever $s>s_{t h}$, and below inelastic thresholds, the unitarity of the $S$ matrix implies

$$
\operatorname{Im} t_{I J}=\sigma_{\alpha \beta}\left|t_{I J}\right|^{2},
$$


where $\sigma_{\alpha \beta}$ is the two particle phase space. Explicitly,

$$
\sigma_{\alpha \beta}(s)=\sqrt{\left(1-\frac{\left(M_{\alpha}+M_{\beta}\right)^{2}}{s}\right)\left(1-\frac{\left(M_{\alpha}-M_{\beta}\right)^{2}}{s}\right)} .
$$

As a consequence of Eq. (4), the partial wave can be parametrized as

$$
t_{I J}(s)=\frac{1}{\sigma_{\alpha \beta}(s)} e^{i \delta_{I J}(s)} \sin \delta_{I J}(s)
$$

and $\delta_{I J}(s)$ is called the $I J$ phase shift.

We have already mentioned that the ChPT amplitudes are obtained as an expansion in external momenta and masses. That is,

$$
t_{I J} \simeq t_{I J}^{(0)}+t_{I J}^{(1)}+t_{I J}^{(2)}+\cdots,
$$

where, for the cases we are interested in, $t_{I J}^{(0)}$ is $O\left(p^{2}\right), t_{I J}^{(1)}$ is $O\left(p^{4}\right)$, etc. In practice, we can only obtain the few first terms of the series above and, therefore, the amplitude only satisfies the unitarity condition perturbatively:

$$
\begin{gathered}
\operatorname{Im} t_{I J}^{(0)}=0, \\
\operatorname{Im} t_{I J}^{(1)}=\sigma_{\alpha \beta} t_{I J}^{(0) 2}, \\
\operatorname{Im}\left(t_{I J}^{(2)}+t_{I J}^{(1)}\right)=\sigma_{\alpha \beta}\left(t_{I J}^{(0) 2}+2 t_{I J}^{(0)} \operatorname{Re} t_{I J}^{(1)}\right) \simeq \sigma_{\alpha \beta}\left|t_{I J}^{(0)}+t_{I J}^{(1)}\right|^{2} .
\end{gathered}
$$

The $O\left(p^{2}\right)$ terms were given by Weinberg [1] and they are called the low-energy theorems. The next order contributions to $\pi \pi$ scattering were given in [2,3]. The calculation for $\pi K$ scattering can be found in [22,23], although we have found that the formulas in the literature do not satisfy Eq. (8). We will comment on that later. Very recently, a complete calculation of the $O\left(p^{6}\right)$ contribution to elastic $\pi \pi$ scattering [5] has appeared. Although we will not use it, we will compare some of its results with those of our method.

\section{THE INVERSE AMPLITUDE METHOD}

\section{A. Derivation from dispersion theory}

Let us briefly review the standard derivation $[7,13]$ of the inverse amplitude method, since we will use it later in order to understand the applicability of the method.

Any partial wave obtained from a relativistic quantum field theory should present a characteristic analytic structure in the complex $s$ plane. Indeed, the reaction threshold becomes a cut in the real axis from $s_{t h}$ to $+\infty$. Because of crossing symmetry, there should be another left cut along the negative axis. If we now apply Cauchy's theorem to our complex amplitudes we obtain integral equations known as dispersion relations. For instance, a three times subtracted dispersion relation is

$$
\begin{aligned}
t_{I J}(s)= & C_{0}+C_{1} s+C_{2} s^{2}+\frac{s^{3}}{\pi} \int_{\left(M_{\alpha}+M_{\beta}\right)^{2}}^{\infty} \frac{\operatorname{Im} t_{I J}\left(s^{\prime}\right) d s^{\prime}}{\left.s^{\prime}-s-i \epsilon\right)} \\
& +I_{\mathrm{LC}}\left(t_{I J}\right),
\end{aligned}
$$

where we have not written explicitly the left cut $\left(I_{\mathrm{LC}}\right)$ contribution. The number of subtractions needed depends on how the amplitude behaves at infinity in order to ensure the vanishing of the contributions coming from closing the integral contour. We have chosen three subtractions since we are going to use $O\left(p^{4}\right) \mathrm{ChPT}$ amplitudes which at high $s$ behave as $s^{2}$. But our arguments remain valid for $O\left(p^{6}\right)$ amplitudes when using four times subtracted dispersion relations, etc.

The ChPT partial waves present both cuts and we can calculate both the subtraction constants $C_{0}, C_{1}, C_{2}$ and the integrand inside Eq. (9) perturbatively:

$$
\begin{gathered}
t_{I J}^{(0)}=a_{0}+a_{1} s, \\
t_{I J}^{(1)}=b_{0}+b_{1} s+b_{2} s^{2}+\frac{s^{3}}{\pi} \int_{\left(M_{\alpha}+M_{\beta}\right)^{2} s^{\prime 3}\left(s^{\prime}-s-i \epsilon\right)}^{\infty} \\
+I_{\mathrm{LC}}\left(t_{I J}^{(1)}\right),
\end{gathered}
$$

where we have expanded the subtraction constants in terms of $M_{\alpha}^{2} / F_{\beta}^{2}$.

The IAM is based on the fact that the function $1 / t_{I J}$ displays the very same analytic structure of $t_{I J}$, apart from some possible pole contributions. For later convenience, we will make use of $G(s)=t_{I J}^{(0) 2} / t_{I J}$. Notice that we have multiplied $1 / t$ by a real function without singularities; thus we keep the same analytic structure and we can write a very similar dispersion relation:

$$
\begin{aligned}
G(s)= & G_{0}+G_{1} s+G_{2} s^{2}+\frac{s^{3}}{\pi} \int_{\left(M_{\alpha}+M_{\beta}\right)^{2} s^{\prime 3}\left(s^{\prime}-s-i \epsilon\right)}^{\infty} \\
& +I_{\mathrm{LC}}(G)+I_{\mathrm{PC}},
\end{aligned}
$$

where $I_{\mathrm{PC}}$ stands for possible pole contributions. The advantage of using $G(s)$ is that, using Eqs. (4) and (8), we can calculate exactly the integral over the right cut (but not on the left, since those equations only hold on the elastic cut), as

$$
\operatorname{Im} G=-t_{I J}^{(0) 2} \frac{\operatorname{Im} t_{I J}}{\left|t_{I J}\right|^{2}}=-t_{I J}^{(0) 2} \sigma=-\operatorname{Im} t_{I J}^{(1)} .
$$

Note that we denote by $t_{I J}$ the exact amplitude, which is unknown, although we know its analytic properties. In contrast, the expressions for $t_{I J}^{(0)}$ and $t_{I J}^{(1)}$, etc., have been calculated explicitly.

As we did before, we can also expand the $G_{i}$ subtraction coefficients in powers of $M_{\alpha}^{2} / F_{\beta}^{2}$, and then rewrite the dispersion relation for $G(s)$, which now reads

$$
\begin{aligned}
\frac{t_{I J}^{(0) 2}}{t_{I J}} \simeq & a_{0}+a_{1} s-b_{0}-b_{1} s-b_{2} s^{2} \\
& -\frac{s^{3}}{\pi} \int_{\left(M_{\alpha}+M_{\beta}\right)^{2} s^{\prime 3}\left(s^{\prime}-s-i \epsilon\right)}^{\infty}-I_{\mathrm{LC}}\left(t_{I J}^{(1)}\right)+I_{\mathrm{PC}} \\
\simeq & t_{I J}^{(0)}-t_{I J}^{(1)},
\end{aligned}
$$

where we have approximated $\operatorname{Im} G \simeq-\operatorname{Im} t_{I J}^{(1)}$ on the left cut and we have neglected the pole contribution. In other words, 


$$
t_{I J} \simeq \frac{t_{I J}^{(0) 2}}{t_{I J}^{(0)}-t_{I J}^{(1)}}
$$

This is the IAM result that we are going to use in the present work. Incidentally, Eq. (14) can be understood as the formal [1,1] Padé approximant of the ChPT amplitude.

It is important to remark that if we expand again Eq. (14) at low energies, we find

$$
t_{I J} \simeq \frac{t_{I J}^{(0) 2}}{t_{I J}^{(0)}-t_{I J}^{(1)}} \simeq t_{I J}^{(0)}+t_{I J}^{(1)}+O\left(p^{6}\right) .
$$

That is, we recover the ChPT expansion. Hence, up to $O\left(p^{6}\right)$ our method and ChPT yield the same low-energy results if the same chiral Lagrangian parameters are used.

\section{B. The applicability of the inverse amplitude method}

Let us review all the approximations made in the previous section, in order to comment how they will constrain the IAM applicability.

\section{The left cut}

In Eq. (13) we have replaced the $G(s)$ left cut integral by that of $-t_{I J}^{(1)}(s)$. As we have remarked in the preceding discussion, Eqs. (4) and (8) are only exact on the right cut. On the left cut we cannot write the chain of equalities that lead to Eq. (12). Nevertheless, if we use the ChPT result as an approximation,

$$
\operatorname{Im} G=-t_{I J}^{(0) 2} \frac{\operatorname{Im} t_{I J}}{\left|t_{I J}\right|^{2}} \simeq-\operatorname{Im} t_{I J}^{(1)}+O\left(p^{6}\right),
$$

we get

$$
\begin{aligned}
I_{\mathrm{LC}}(G) & =\int_{-\infty}^{0} \frac{\operatorname{Im} G_{I J}\left(s^{\prime}\right) d s^{\prime}}{s^{\prime 3}\left(s^{\prime}-s-i \epsilon\right)} \simeq-\int_{-\infty}^{0} \frac{\operatorname{Im} t_{I J}^{(1)}\left(s^{\prime}\right) d s^{\prime}}{\left.s^{\prime}-s-i \epsilon\right)} \\
& =-I_{\mathrm{LC}}\left(t_{I J}^{(1)}\right)
\end{aligned}
$$

Notice that, in order to obtain the $I J$ phase shifts, we are going to calculate $t_{I J}(s)$ for real $s>4 M_{\pi}$. That means that the denominator $\left(s^{\prime}-s-i \epsilon\right)$ inside the integrals is never going to be very small, which somehow will wash out the error on the left cut. But note also that treating differently the right and left cuts violates crossing symmetry.

Indeed, in [24] it has already been pointed out that the Padé approximants do not reproduce correctly the subleading logarithms that would appear at next order in the chiral expansion $\left[O\left(p^{6}\right)\right.$ in this case]. Of course, they would be obtained if we applied the IAM to the chiral amplitudes at $O\left(p^{6}\right)$, but still the method would not yield the correct logarithms at $O\left(p^{8}\right)$ and so on. At high energies chiral logarithms are not so relevant, but at low energies they are a very important feature of ChPT and indeed they can give the dominant contribution in some channels.

Nevertheless, from Eq. (15) we see that at low energies the IAM yields the very same $O\left(p^{4}\right)$ ChPT expansion, including the dominant chiral logarithms. The contribution from the left cut and subleading logarithms is $O\left(p^{6}\right)$. As a consequence, if we try to make a low-energy fit to the data, the parameters that we would obtain with the IAM would not lie very far from those of ChPT, but they will not be the same. That is the reason why, in the following sections, we will denote with a hat the parameters obtained from any IAM fit. ${ }^{1}$

\section{Resonances and the pole contribution}

In Eq. (13), we have neglected the contributions coming from zeros in the amplitude, that will appear as poles of the inverse function. There is no way to know a priori whether or not a partial wave will vanish for a given value of $s$, although it is known that chiral amplitudes have zeros below threshold, which are known as Adler zeros. Their position is not known except for the $I=1, J=1$ channel, where the pole is located at threshold. In our derivation it is compensated by the same zero in the $t_{11}^{(0)}$ channel. That is not the case for the $J=0$ amplitudes and, therefore, we are neglecting the contribution of their residue. Consequently, our amplitudes are not valid to obtain Adler zeros and that will affect our results at low energy [but no more than $O\left(p^{6}\right)$ ]. That is another reason to differentiate the parameters obtained from our fit from those of the pure chiral expansion (see footnote 1).

\section{Multiplying by $t_{I J}^{(0)}$}

This is apparently a harmless assumption in the above reasoning, although it dramatically affects the results of the IAM. In fact, it can happen that $t_{I J}^{(0)}=0$. In the $(I, J)=(0,0),(1,1),(2,0)$ channels of $\pi \pi$ scattering or in the $(3 / 2,0),(1 / 2,0),(1 / 2,1)$ in $\pi K$, this only occurs for isolated values of $s$, at or below threshold. In particular, that means that the IAM amplitudes will have the same zeros as the lowest order chiral amplitudes. However, every other partial wave vanishes at $O\left(p^{2}\right)$, for any $s$. As a consequence, the formula in Eq. (14) is no longer valid.

Nevertheless, we can generalize our previous derivation, in order to include those channels whose leading order is $O\left(p^{4}\right)$. We only have to go through the very same steps, although now we would write a dispersion relation for $t_{I J}^{(2)}$. But let us remember that the main improvement of the approach is that we are calculating exactly the integral of $\operatorname{Im} G(s)$ over the right cut. However, for that purpose we need an imaginary part, and by looking at Eq. (8) we can see that $t_{I J}^{(0)}=0$ implies that $\operatorname{Im} t_{I J}^{(1)}=\operatorname{Im} t_{I J}^{(2)}=0$. Therefore, unless we have a calculation up to $O\left(p^{8}\right)$, the corresponding imaginary part will vanish. Hence, when following the derivation of the IAM if $t_{I J}^{(0)}=0$ the best we can get is plain ChPT again. At present, only $O\left(p^{6}\right)$ calculations are available and we can only expect to obtain a real improvement with our approach in the six channels listed above. Thus, we will not be able to reproduce the $f_{2}(1200)$ resonance.

\footnotetext{
${ }^{1}$ While we were revising this paper a work by M. Boglione and M. R. Pennington [48] appeared in which they propose other schemes with better approximations to the left cut and also include possible contributions from Adler zeros.
} 


\section{Elastic unitarity}

In order to obtain $\operatorname{Im} G$ on the right cut, Eq. (12), we have just made use of the elastic unitarity condition of Eq. (4). However, the right cut is composed of many superimposed cuts, each one corresponding to a different inelastic intermediate channel. Actually, Eq. (4) is only true below the first inelastic threshold, and the real unitarity condition reads

$$
\operatorname{Im} t_{\alpha \beta \rightarrow \alpha \beta}=\sum_{A} \sigma_{A}\left|t_{\alpha \beta \rightarrow A}\right|^{2} \Theta\left(s-s_{A}\right)
$$

The sum is over all the physically accessible intermediate states $A$, whose phase space is $\sigma_{\alpha \beta}$.

As far as we are neglecting electromagnetic interactions, the first inelastic channel in $\pi \pi$ is the four pion intermediate state, at $550 \mathrm{MeV}$. Similarly, for $\pi K$ is $\pi K \pi \pi$, whose threshold is $\simeq 910 \mathrm{MeV}$. Strictly speaking, the elastic approximation is exact only for lower energies. Nevertheless, the contribution of these intermediate states is strongly suppressed by the four particle phase space and we expect the IAM to provide a good approximation.

Unfortunately, within the range of energies we are interested in, there are intermediate channels which are not sup- pressed by phase space. Indeed, at approximately $985 \mathrm{MeV}$ the inelastic $K \bar{K}$ threshold opens up. Its phase space factor is the $\sigma_{\alpha \beta}$ in Eq. (5), with $M_{\alpha}=M_{\beta}=M_{K}$. Therefore, above the two kaon threshold we have to reconsider the derivation of the IAM. Let us illustrate with $\pi \pi$ scattering how inelastic effects modify our result.

As the starting point, for $s>s_{K} \bar{K}$, we have a new unitarity relation:

$$
\operatorname{Im} t=\sigma_{\pi \pi}|t|^{2}+\sigma_{K \bar{K}}\left|t_{K}\right|^{2}
$$

where we have denoted by $t$ the generic $t_{I J}$ pion elastic scattering amplitude and by $t_{K}$ the $I J$ partial wave of the process $\pi \pi \rightarrow K \bar{K}$. Thus we now have, for $s>s_{K \bar{K}}$, that

$$
\operatorname{Im} G=-t_{I J}^{(0) 2} \frac{\operatorname{Im} t_{I J}}{\left|t_{I J}\right|^{2}}=-t_{I J}^{(0) 2}\left(\sigma_{\pi \pi}+\sigma_{K \bar{K}} \frac{\left|t_{K}\right|^{2}}{|t|^{2}}\right)
$$

which differs from Eq. (12) in the term coming from two kaon intermediate production. If we follow the very same steps of our previous derivation, we arrive at

$$
\frac{t^{(0) 2}}{t_{I J}} \simeq t^{(0)}-t^{(1)}-\frac{s^{3}}{\pi} \int_{4 M_{\pi}^{2}}^{\infty} \frac{\sigma_{K \bar{K}}}{s^{\prime 3}\left(s^{\prime}-s-i \epsilon\right)} \underbrace{\left(t^{(0) 2}\left(s^{\prime}\right) \frac{\left|t_{K}\left(s^{\prime}\right)\right|^{2}}{\left|t\left(s^{\prime}\right)\right|^{2}}-t_{K}^{(0) 2}\left(s^{\prime}\right)\right)}_{\Delta\left(s^{\prime}\right)} d s^{\prime}
$$

Notice that, using ChPT, $\Delta\left(s^{\prime}\right) \simeq 0+O\left(p^{6}\right)$. But at these high energies that is not negligible. In addition, we are interested in the above integral for physical values of $s$ and, therefore, the denominator will be almost divergent for some $s^{\prime}$. For these reasons we cannot neglect this integral and then we should not trust the IAM since it could miss some relevant physical features.

That is indeed the case in pion scattering since, as it can be seen in Table I, there is one resonance, $f_{0}(980)$, whose nature is closely related to the $K \bar{K}$ threshold. Nowadays, the interpretation of that resonance is still controversial: different authors propose different poles (not always just one) in the vicinity of the $K \bar{K}$ inelastic cut $[26,27]$. As we will see later, our approach is not able to reproduce any of these poles, which is consistent with the fact that the IAM makes use just of elastic unitarity.

At this point, we want to remark on the importance of understanding why and when the method no longer yields the right results. Let us remember that we are also thinking of possible applications of this unitarization procedures to the electroweak chiral effective Lagrangian, whose reference model is the standard model with a heavy Higgs boson. In such a case, one would expect to see a broad resonance in the scalar channel and we want to have a unitarization procedure whose predictions we can trust.

\section{5. $O\left(p^{4}\right)$ approximation}

Throughout the derivation of the IAM, we have been using the chiral amplitudes up to $O\left(p^{4}\right)$. Nevertheless, it is possible to extend the argument to include higher order terms, as, for instance, the $O\left(p^{6}\right)$ contributions. In that case we would have started from a four times subtracted dispersion relation for the two-loop calculation. Once more, the integral over the right cut would be related to the one for $G(s)=t_{I J}^{(0) 2} / t_{I J}$. Working out the expansion of the subtraction constants, we would then arrive at

$$
t_{I J} \simeq \frac{t_{I J}^{(0) 2}}{t_{I J}^{(0)}-t_{I J}^{(1)}+t_{I J}^{(1) 2} / t_{I J}^{(0)}-t_{I J}^{(2)}} .
$$

Again, that is the formal [1,2] Padé approximant, and it satisfies the elastic unitarity condition.

As we have already mentioned, two recent papers have appeared with $O\left(p^{6}\right)$ calculations of $\pi \pi$ scattering within $\mathrm{SU}(2) \mathrm{ChPT}[4,5]$. We have not used these results, since, as we have just seen, they will not help us to overcome any of the preceding objections to the IAM. However, it is quite likely that, had we used them, the parameters of the fits that we will present in the next sections would have been slightly modified. 
TABLE II. Sets of parameters and methods used in the text. Those in the first two columns come from $K_{l 4}$ decays [29]. Those in the third, from data on $K_{l 4}$ and $\pi \pi$ together with some unitarization procedure (Ref. [30]). $M_{\rho}$ is calculated with the central values.

\begin{tabular}{lccc}
\hline \hline Method & $\bar{l}_{1}$ & $\bar{l}_{2}$ & $M_{\rho}$ \\
\hline ChPT & $-0.62 \pm 0.94$ & $6.28 \pm 0.48$ & No resonances \\
\hline Inverse & $-0.62 \pm 0.94$ & $6.28 \pm 0.48$ & $715 \mathrm{MeV}$ \\
amplitude & $-1.7 \pm 1.0$ & $6.1 \pm 0.5$ & $675 \mathrm{MeV}$ \\
\hline \hline
\end{tabular}

\section{IV. $\pi \pi$ SCATTERING IN SU(2) ChPT}

The inverse amplitude method was first applied $[8,15]$ to $\pi \pi$ scattering without the strange quark. In that case, the massless limit displays spontaneous symmetry breaking from $\mathrm{SU}(2)_{L} \times \mathrm{SU}(2)_{R}$ to $\mathrm{SU}(2)_{L+R}$, which is nothing but the usual isospin. The $O\left(p^{4}\right)$ expression for $\pi \pi$ scattering was obtained in $[2,28]$, and it is written in terms of four phenomenological parameters $\bar{l}_{1}, \bar{l}_{2}, \bar{l}_{3}, \bar{l}_{4}$, as well as the mass and pion decay constants, $M_{\pi}$ and $F_{\pi}$. In this section we will review how the method is able to reproduce the $\rho$ resonance. We will show some results for recently proposed new parameters in order to test the IAM predictive power, but we will also present a unitarized fit to the data. We will use not only the $J=0$ phase shifts, but also those with $J=2$, in order to obtain the best fit with the IAM. In this calculation, we have also estimated the error bars of the unitarized parameters.

\section{A. Results using low-energy parameters}

Let us now illustrate what happens if we apply the IAM on the ChPT amplitudes using the chiral parameters obtained from low-energy experiments. We want to see quantitatively to what extent the main physical features are reproduced.

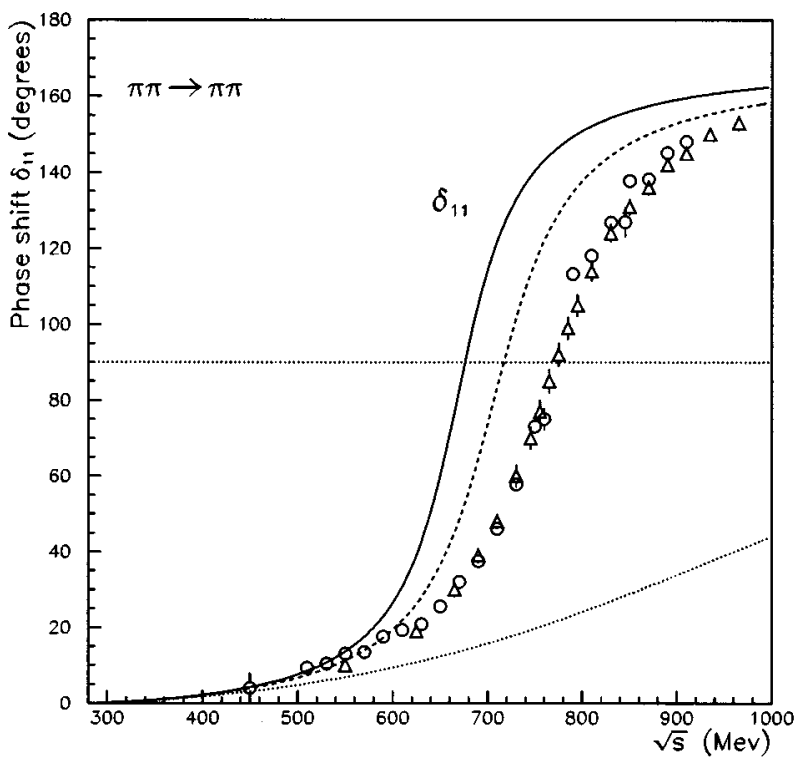

In order to simplify the comparison with previous works, we have chosen $M_{\pi}=139.57 \mathrm{MeV}$ and $F_{\pi}=93.1 \mathrm{MeV}$. The values of the chiral parameters are not so clear, since they have considerable error bars. In Table II we have listed the different sets of parameters that we have taken from the literature to obtain Fig. 1.

Let us remark at this point that for the ChPT phase shifts we are using the definition $\delta \simeq \sigma\left(t^{(0)}+\operatorname{Re} t^{(1)}\right)$ suggested in [24]. Of course, ChPT is just a low-energy approach, but incidentally, these phase shifts coincide with those obtained from the $K$-matrix unitarization defined as

$$
t^{K}=\frac{t^{(0)}+\operatorname{Re} t^{(1)}}{1-i \sigma\left(t^{(0)}+\operatorname{Re} t^{(1)}\right)} .
$$

It can be easily verified that $t^{K}$ satisfies elastic unitarity, Eq. (4), exactly. Consequently, the dotted lines in Fig. 1 not only give the ChPT predictions, but also the results of the $K$-matrix unitarization. We will thus confirm that such a method is not able to reproduce resonances by itself. They have to be added by hand.

In Fig. 1 it can be clearly seen, in the $I=1, J=1$ channel, that the IAM yields a $\rho$-like resonance. The value of its mass is obtained from the point where $\delta=90^{\circ}$ and it lies $10 \%$ to $15 \%$ away from its real value. In this way, the existence of the $\rho$ resonance can be regarded as a prediction of the IAM with ChPT and the parameters obtained from some lowenergy data.

It is also evident that the fit of the $I=2, J=0$ channel is correct up to much higher energies. In Table II we have also included the values of $M_{\rho}$ corresponding to each choice of parameters. For all the cases we have set $\bar{l}_{3}=2.9$ and $\bar{l}_{4}=4.3$ following Ref. [2].

The only feature of $\pi \pi$ scattering that is evidently missing from the unitarized results is the $f_{0}(980)$ resonance in

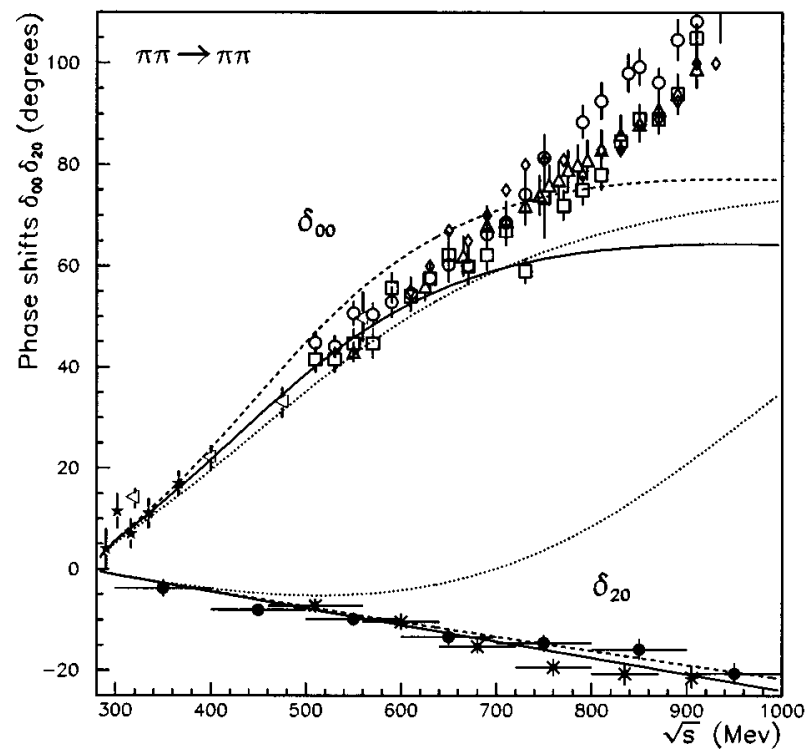

FIG. 1. Phase shifts for $\pi \pi \rightarrow \pi \pi$. The dotted curve is plain ChPT with the $\bar{l}_{i}$ in the first column of Table II. The other two curves are both the result of the IAM: the dashed one has been calculated again with the same parameters whereas the continuous one corresponds to the $\bar{l}_{i}$ in the third column of Table II. The data come from [31] $\left.\triangle \triangle\right),[32](\diamond, \square),[33](\times),[34](\bigcirc),[35](\triangleleft),[36](\star)$, and [37] $(\bullet)$. The results with SU(3) ChPT would have been exactly superimposed on these curves. The straight line stands at $\delta=90^{\circ}$. 

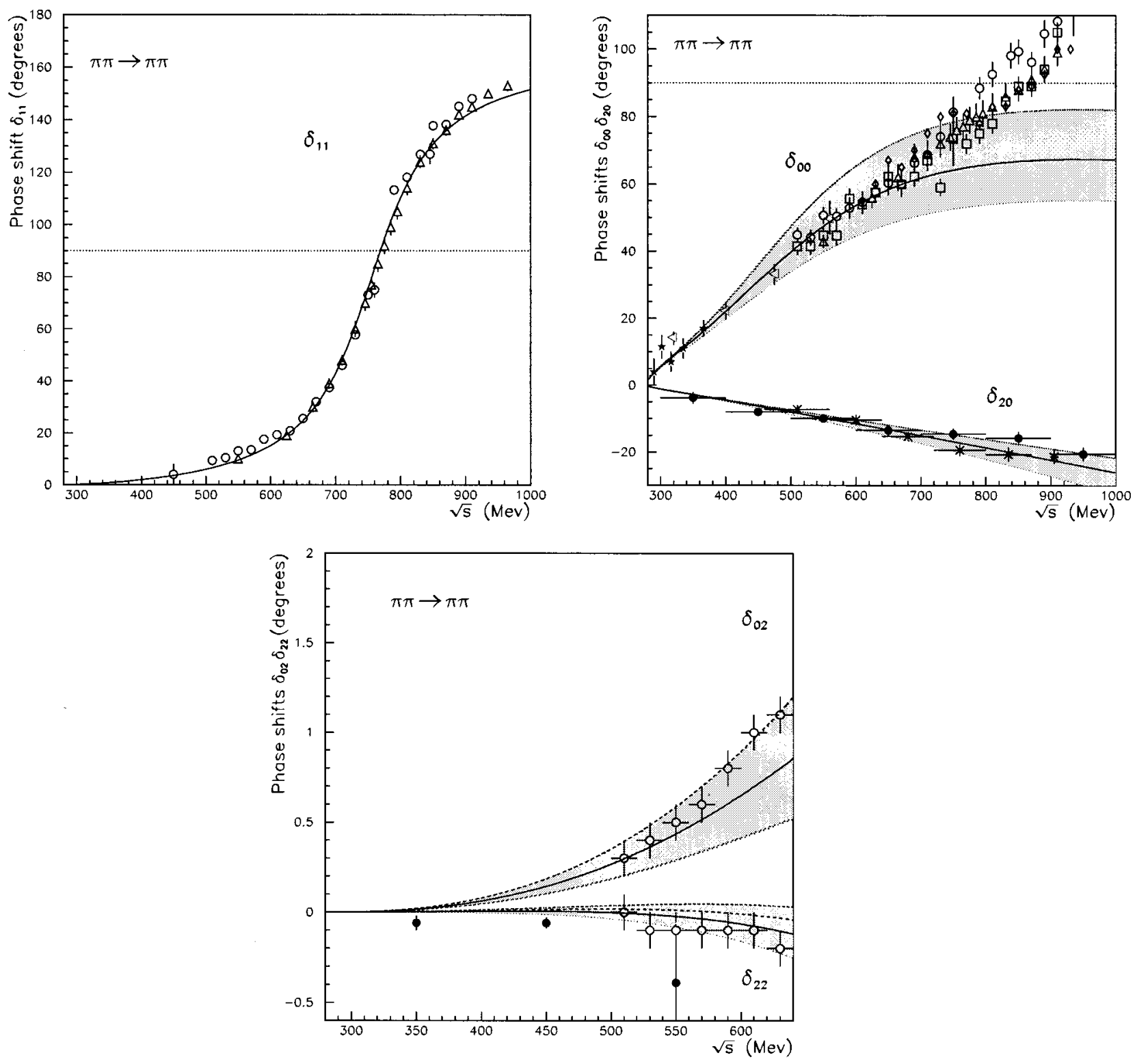

FIG. 2. Pion elastic scattering phase shifts $\delta_{I J}$ obtained from the IAM fit to the correct $M_{\rho}$. The shaded areas cover the error bars of the fitted parameters with the constraint $\hat{l}_{1}-\hat{l}_{2}=-5.95 \pm 0.02$. The dotted straight lines stand at $\delta=90^{\circ}$. Remember that the $J=2$ partial waves have to be calculated as in plain ChPT. Indeed, the dashed lines in those channels correspond to plain ChPT data with the parameters in the first row of Table II. The symbols for the experimental data are the same as in Fig. 1. The corresponding curves within SU(3) ChPT would almost superimpose.

the $I=0, J=0$ channel. In the previous section we saw that this fact is connected with the failure of the whole approach to reproduce the kaon inelastic cut. But let us first obtain a better fit to the data.

\section{B. Unitarized fit}

Now that we have an amplitude that describes the right cut, while keeping at the same time the correct polynomial form from ChPT, it seems natural to use $M_{\rho}[8,15]$ to fit the data. Note that fixing the correct mass does not imply a good fit. For instance, we could get a wrong width. In order to differentiate the parameters thus obtained from those coming from plain ChPT we will call them $\hat{l}_{1}, \hat{l}_{2}$.

The $(1,1)$ channel is almost only sensible to $\bar{l}_{1}-\bar{l}_{2}$. With $\hat{l}_{1}-\hat{l}_{2}=-5.95 \pm 0.02$ we get the $M_{\rho}$ listed in Table I and in Fig. 2 it can be seen that the results are remarkably successful. Later, we will show that we also get the right width.

Once that difference is fixed, we just have to determine one parameter, say $\hat{l}_{2}$. In previous studies $[8,15]$, the unitarized fit to the other phase shifts was used in order to estimate the values of $\hat{l}_{1}$ and $\hat{l}_{2}$. But, as we commented above, the data in the $(0,0)$ channel are not as good as that of $(1,1)$. The same happens for the $(2,0)$ channel, where the curves are not very sensible to small variations in the $\hat{l}_{i}$ parameters. Therefore, in the present work, we have also used the $J=2$ channels (mainly that with $I=0$ ) to further constrain the parameter range. Let us remember now that in these channels $t_{I 2}^{(0)}=0$ and, as we have already discussed in Sec. II, the IAM leads again to plain ChPT. That is why we will only use for 
TABLE III. Parameters and results of the one-loop IAM when $M_{\rho}$ is fixed to its actual value.

\begin{tabular}{lllll}
\hline \hline Method & $\hat{l}_{1}$ & $\hat{l}_{2}$ & $M_{\rho}$ (input) & $\Gamma_{\rho}$ \\
\hline
\end{tabular}

Inverse

amplitude $-0.5 \pm 0.65 .4 \pm 0.6768 .8 \pm 1.1 \mathrm{MeV} 155.6 \pm 1.8 \mathrm{MeV}$

them data up to $\simeq 600 \mathrm{MeV}$, although in other channels we are using data at higher energies.

Thus, the values given in Table III are just a conservative estimate of the range where we obtain a reasonable fit in the $(I, J)=(0,0),(2,0),(0,2)$, and $(2,2)$ channels, when $M_{\rho}$ fixed to its actual value. The results are shown in Fig. 2, where the continuous line corresponds to the central $\hat{l}_{i}$ values and the shaded area to their uncertainties. Notice that the shaded area has always been obtained by varying $\hat{l}_{2}$ within its estimated error.

In Fig. 2 it can be seen how it is not possible to fit the $f_{0}(980)$ resonance with $\mathrm{SU}(2) \mathrm{ChPT}$ and the IAM. It is clear that, even though the actual value of the $\delta_{00}$ phase shift may not lie very far from the unitarized prediction, the qualitative behavior of the curves in this channel is not correct above $800 \mathrm{MeV}$.

With the $\hat{l}_{i}$ fit we can obtain the total Breit-Wigner width of the $\rho$ resonance from

$$
\Gamma_{\rho}=\frac{M_{\rho}^{2}-s}{M_{\rho}} \tan \delta_{11}(s) .
$$

Indeed we have computed it for different values of $s$ around $M_{\rho}^{2}$. The result is given in Table III and it is quite close to the experimental result (see Table I), although slightly higher. We will see that it is possible to obtain the right value when using SU(3) ChPT.

As we have already commented, this result is not at all trivial, since fitting the right mass does not ensure a correct description of the resonance. Therefore, even though we are now using the $M_{\rho}$ experimental value, the $\Gamma_{\rho}$ width is again a prediction of the IAM. In contrast, in a unitarization scheme where one introduces the resonances by hand, one has to give both the masses and the widths.

\section{SU(3) CHIRAL PERTURBATION THEORY}

The extension of the ChPT approach to include the strange quark was done, once more, by Gasser and Leutwyler [3]. In this case there are eight Goldstone bosons, which are identified with the three pions, the four kaons, and the $\eta$. In principle, it is possible to calculate the amplitudes of any process involving any combination of these particles. But the thresholds for these reactions are much higher than in pion scattering, which in practice restricts severely the effectiveness of the approach.

Nevertheless, the lowest two particle threshold apart from $\pi \pi$ scattering is that of $\pi K$ elastic scattering at $630 \mathrm{MeV}$, which is still within the applicability range of ChPT. The calculation of this amplitude to $O\left(p^{4}\right)$ was performed by Bernard, Kaiser, and Meissner [22,23] who also gave the $O\left(p^{4}\right)$ result for $\pi \pi$ within $\mathrm{SU}(3) \mathrm{ChPT}$. In the literature, these formulas have sometimes appeared with some minor errata which have been corrected in the DAФNE physics handbook [25]. However, even those formulas do not satisfy perturbative unitarity (see the Appendix). Following the work in [22], we have rederived an expression which does satisfy that requirement, and we have included it in the Appendix, together with a discussion on how it is obtained and its unitarity properties.

In the $\mathrm{SU}(3)$ case there are more phenomenological parameters that we have set to

$$
\begin{gathered}
M_{K}=493.65 \mathrm{MeV}, \quad M_{\eta}=548.8 \mathrm{MeV}, \\
F_{K}=1.22 F_{\pi}, \quad F_{\eta}=1.3 F_{\pi} .
\end{gathered}
$$

There are also 12 one-loop parameters, denoted by $L_{i}^{r}(\mu)$. However, only $L_{1}^{r}, L_{2}^{r}, L_{3}, L_{4}^{r}, L_{5}^{r}, L_{6}^{r}$, and $L_{8}^{r}$ appear in $\pi K$ scattering, whereas in pion scattering only the following combinations are present:

$$
\begin{gathered}
2 L_{1}^{r}+L_{3}, \quad L_{2}^{r}, \\
2 L_{4}^{r}+L_{5}^{r}, \quad 2 L_{6}^{r}+L_{8}^{r} .
\end{gathered}
$$

Again, and in order to simplify the comparison with previous works, we have fixed the values [3]

$$
\begin{array}{cl}
L_{4}^{r}\left(M_{\eta}\right)=0, & L_{5}^{r}\left(M_{\eta}\right)=0.0022, \quad L_{6}^{r}\left(M_{\eta}\right)=0, \\
& L_{8}^{r}\left(M_{\eta}\right)=0.0011 .
\end{array}
$$

A precise value of these parameters is not very important since they are related to the different masses and decay constants that we had already fixed. Hence, in practice, the only relevant parameters for $\pi \pi$ and $\pi K$ scattering in $\mathrm{SU}(3)$ are $L_{1}^{r}, L_{2}^{r}$, and $L_{3}$.

The IAM was first applied to SU(3) ChPT in [13], where we showed that it reproduces not only the $\rho(770)$ resonance but also the $K^{*}(892)$. Our aim in this section is first to study the predictive power of the method, whether it can accommodate further resonant states, or why it cannot. Then, we will present a simultaneous fit to $\pi \pi$ and $\pi K$ scattering to the $\rho$ and $K^{*}$ masses. The new feature of this analysis is that it uses the corrected ChPT expressions for $\pi K$ scattering which now satisfy perturbative unitarity (see the Appendix) and the fact that we also use the data on the $J=2$ in $\pi \pi$ scattering channels. We will also estimate the error bars on the best fit that will be used to obtain numerical values for some interesting phenomenological quantities. This fit will also allow us, in Sec. VI, to perform a numerical study of the analytic structure of the IAM amplitudes in the complex $s$ plane.

\section{A. Results using low-energy parameters}

Let us then start with the IAM using parameters obtained from low-energy data. In Table IV we list different choices of parameters and methods together with their results for the $\rho$ and $K^{*}$ masses. As in the case of SU(2) ChPT, the IAM is able to predict from low-energy data the existence of both resonant states. Remarkably, the masses thus obtained lie again $10 \%$ to $15 \%$ away from their actual values. 
TABLE IV. Different sets of parameters and methods used in the text. Those of the first two columns come from $K_{l 4}$ decays [29]. Those of the third come from data on $K_{l 4}$ and $\pi \pi$ together with some unitarization procedure (for details see Ref. [30]). The quoted values of $M_{\rho}$ and $M_{K^{*}}$ are calculated with the central values.

\begin{tabular}{lccccc}
\hline \hline Method & $L_{1}^{r}\left(M_{\eta}\right) \times 10^{3}$ & $L_{2}^{r}\left(M_{\eta}\right) \times 10^{3}$ & $L_{3} \times 10^{3}$ & $M_{\rho}$ & $M_{K^{*}}$ \\
\hline ChPT & $0.65 \pm 0.28$ & $1.89 \pm 0.26$ & $-3.06 \pm 0.92$ & & \\
\hline Inverse & $0.65 \pm 0.28$ & $1.89 \pm 0.26$ & $-3.06 \pm 0.92$ & $717 \mathrm{MeV}$ & $847 \mathrm{MeV}$ \\
amplitude & $0.6 \pm 0.3$ & $1.75 \pm 0.3$ & $-3.5 \pm 1.1$ & $680 \mathrm{MeV}$ & $804 \mathrm{MeV}$ \\
\hline \hline
\end{tabular}

In Fig. 3 we show the result of applying the IAM to $\pi K$ scattering, with the parameters given in Table IV. In contrast with plain $O\left(p^{4}\right)$ ChPT (or the $K$-matrix unitarization method, since they yield the same phase shifts), it is evident that the IAM not only accommodates the $K^{*}$ resonance, but it also reproduces the $(3 / 2,0)$ channel.

We do not display the results for $\pi \pi$ scattering in SU(3) because they will almost superimpose with those in Fig. 1. Indeed, the $\bar{l}_{i}$ parameters in rows 2 and 4 of Table II were obtained, respectively, from the $L_{1}^{r}, L_{2}^{r}, L_{3}$ in rows 2 and 4 of Table IV $[29,30]$, by means of

$$
\begin{gathered}
\bar{l}_{1}=96 \pi^{2}\left(4 L_{1}^{r}\left(M_{\eta}\right)+2 L_{3}-\frac{\nu_{K}}{24}-\frac{\nu_{\pi}}{3}\right), \\
\bar{l}_{2}=48 \pi^{2}\left(4 L_{2}^{r}\left(M_{\eta}\right)-\frac{\nu_{K}}{12}-\frac{2 \nu_{\pi}}{3}\right), \\
\nu_{\alpha}=\frac{1}{32 \pi^{2}} \ln \left(\frac{M_{\alpha}^{2}}{M_{\eta}^{2}}\right), \quad \alpha=\pi, K .
\end{gathered}
$$

As a matter of fact, we have calculated independently the $\pi \pi$ elastic scattering in $\mathrm{SU}(2)$ and $\mathrm{SU}(3)$. Using the above equations to relate the parameters in both cases, and below

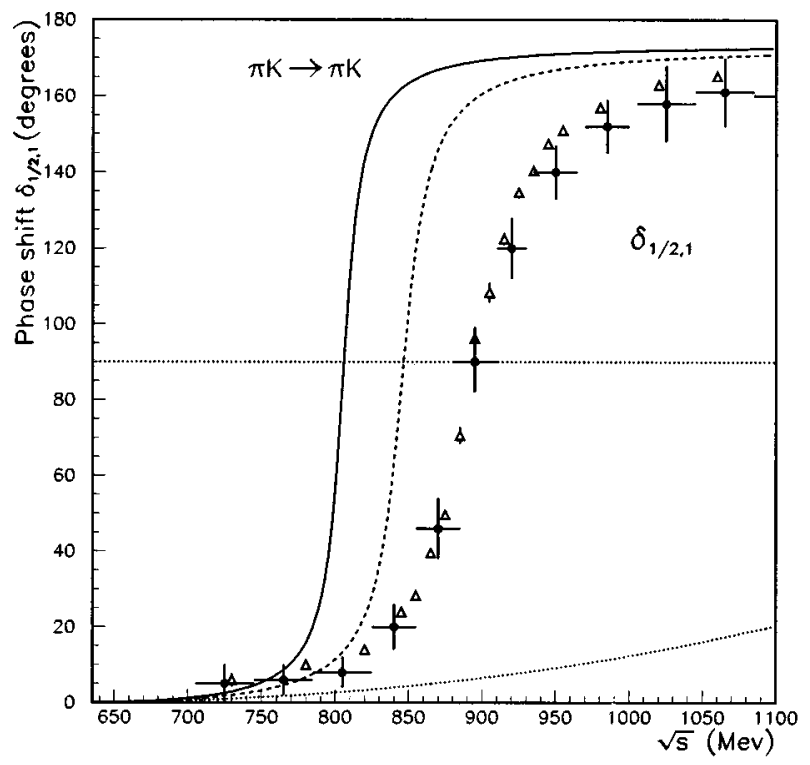

kaon threshold, we have obtained the same results up to numerical differences $(\simeq 1 \%)$, which would be unobservable in the figures. That is a nice check of our programs. Therefore, Fig. 1 is also the result for $\pi \pi$ scattering in the SU(3) formalism, but now with the parameters in Table IV.

\section{B. Unitarized fit}

Again, we have an expression for the amplitude that behaves correctly with respect to unitarity and that presents the right form in the low-energy limit. Therefore, we can try to use the actual $\rho(770)$ and $K^{*}(892)$ masses in order to fit the $\pi \pi$ and $\pi K$ phase shifts. We remark once more that nothing ensures that fitting the right masses will give us the right description, since, among other things, the widths of the resonances could be wrong.

When dealing with the SU(3) chiral Lagrangian we have more parameters, and the way they appear in the amplitudes is more complicated. Let us first start with the $\pi \pi$ scattering partial waves in SU(3). As we have commented in Sec. III B 1, in order to avoid confusions with the ChPT lowenergy parameters, we will denote the parameters of our fit by $\hat{L}_{i}^{r}$.

The $(1,1)$ channel only depends on $2 L_{1}^{r}+L_{3}-L_{2}^{r}$, and will be fixed with $M_{\rho}$. In so doing we get

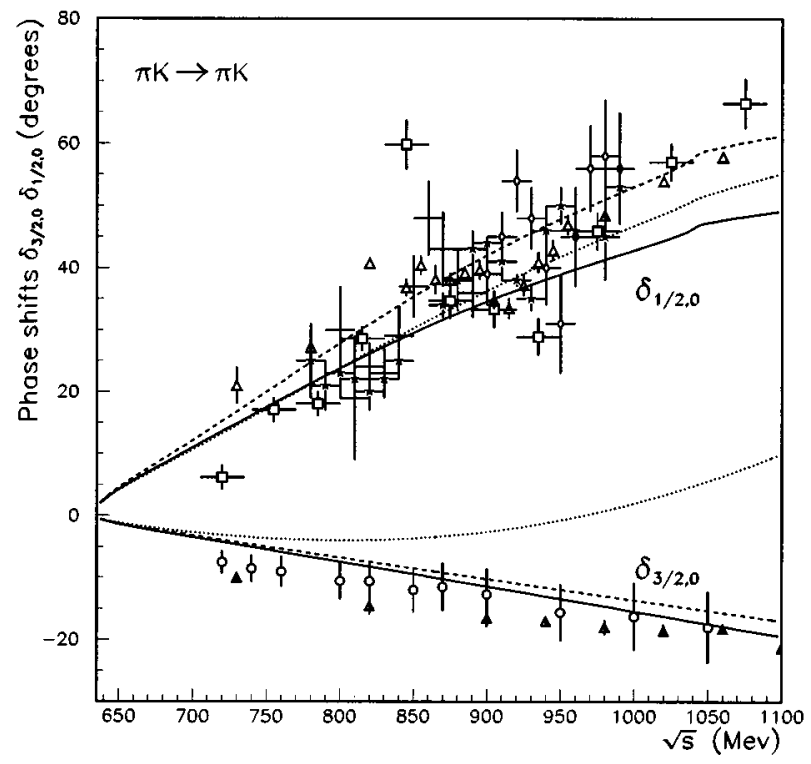

FIG. 3. Phase shifts for elastic $\pi K$ scattering. The dotted curve is plain ChPT with the $L_{i}$ parameters in the first column of Table IV. The other two curves are both obtained from the IAM: the dashed one again with the same parameters and the continuous one with those in the third column of Table 4. The experimental data come from [38] $(\bullet),[39](\star),[40](\bigcirc),[41](\diamond),[42](\square)$, and [43] $(\triangle)$. The straight dotted line stands at $\delta=90^{\circ}$. 
TABLE V. Parameters and results of the SU(3) IAM, when $M_{\rho}=768.8 \pm 1.1 \mathrm{MeV}$ and $\bar{M}_{K^{*}}=894.00 \pm 2.5 \mathrm{MeV}$, are fixed to their actual values. Notice that for $K^{*}(892)$ we have chosen an average mass between its different charge states.

\begin{tabular}{lccccc}
\hline \hline Method & $\hat{L}_{1}^{r}\left(M_{\eta}\right) \times 10^{3}$ & $\hat{L}_{2}^{r}\left(M_{\eta}\right) \times 10^{3}$ & $\hat{L}_{3} \times 10^{3}$ & $\Gamma_{\rho}$ & $\Gamma_{K^{*}}$ \\
\hline $\begin{array}{l}\text { Inverse } \\
\text { amplitude }\end{array}$ & $0.41 \pm 0.20$ & $1.48 \pm 0.33$ & $-2.44 \pm 0.21$ & $149.9 \pm 1.2 \mathrm{MeV}$ & $41.2 \pm 1.9 \mathrm{MeV}$ \\
\hline \hline
\end{tabular}

$$
2 \hat{L}_{1}^{r}+\hat{L}_{3}-\hat{L}_{2}^{r}=(-3.11 \pm 0.01) \times 10^{-3}
$$

As a consistency check we see that it is within a $1 \%$ of $-3.14 \times 10^{-3}$ which is obtained from the $\hat{l}_{i}$ parameters of the $\mathrm{SU}(2)$ case, with the help of Eq. (29).

Once again, we use the channels $(I, J)=(0,0),(2,0),(0,2)$, and $(2,2)$ to determine the best $\hat{L}_{2}^{r}$ value, which indeed is the same that we would have obtained from the $\hat{l}_{2} \mathrm{SU}(2)$ parameter by means of Eq. (29). It can be found in Table V. Hence, the best $\mathrm{SU}(3)$ fit of the $\pi \pi$ phase shifts yields almost the same results as those obtained with $\mathrm{SU}(2)$ and the very same Fig. 2 remains valid for $S U(3)$. Nevertheless, when computing the $\Gamma_{\rho}$ within the $S U(3)$ formalism, we obtain a much better value than in $\mathrm{SU}(2)$, which was about $5 \mathrm{MeV}$ too high. It is also listed in Table $\mathrm{V}$.

Finally, we will use $\hat{L}_{3}$ to fix the correct $K^{*}(892)$ mass. However, the $K^{*}(892)$ has an added subtlety, namely, that the mass splitting between different charge states is of the order of $5 \mathrm{MeV}$. This is a small isospin-breaking effect that we have not included in our approach. Therefore, we have used an average mass $\bar{M}_{K^{*}}=894.0 \pm 2.5 \mathrm{MeV}$ with an error bar that includes the mass of any $K^{*}(892)$ state, no matter what its charge may be. That uncertainty has also been taken into account in the $\hat{L}_{i}^{r}$ error estimates.

Once we have $\hat{L}_{3}$, we use $\hat{L}_{2}^{r}$ and Eq. (30) to obtain $\hat{L}_{1}^{r}$. The parameters of this fit have been collected in Table $\mathrm{V}$,

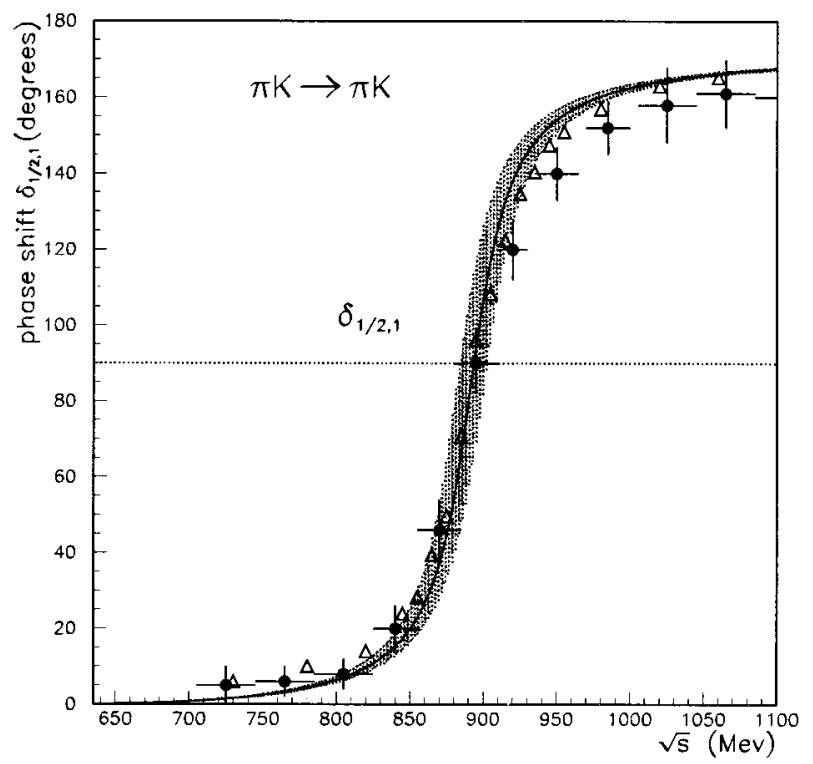

together with $\Gamma_{\rho}$ and $\Gamma_{K^{*}}$, which can be considered as predictions of the approach. Notice, however, that in this case the width of the $K^{*}(892)$ resonance lies $20 \%$ away from its actual value, which, nevertheless, is a reasonably good result in view of the whole fit in that channel.

Concerning the $\hat{L}_{i}$ parameters, they are compatible with those in Table IV, which were obtained from low-energy data. Even more, they are also consistent with other parameters obtained from the IAM applied to the form factors of the $K \rightarrow \pi \pi l \nu$ decays [14], which are very well known experimentally:

$$
\begin{aligned}
& \hat{L}_{1}^{r}\left(M_{\eta}\right)=(0.74 \pm 0.14) \times 10^{-3} \\
& \hat{L}_{2}^{r}\left(M_{\eta}\right)=(1.07 \pm 0.18) \times 10^{-3} \\
& \hat{L}_{3}\left(M_{\eta}\right)=(-2.45 \pm 0.52) \times 10^{-3}
\end{aligned}
$$

(notice that in that reference they are using $F_{K}=F_{\pi}$, so that the parameters do necessarily differ).

Nevertheless, it would not make any sense to try to reduce the error bars of these parameters. We consider that the approach that we have been following here can only be consistent within a few percent error level. In order to have a better accuracy it would be necessary to take into account higher order ChPT corrections, isospin-breaking effects, and the

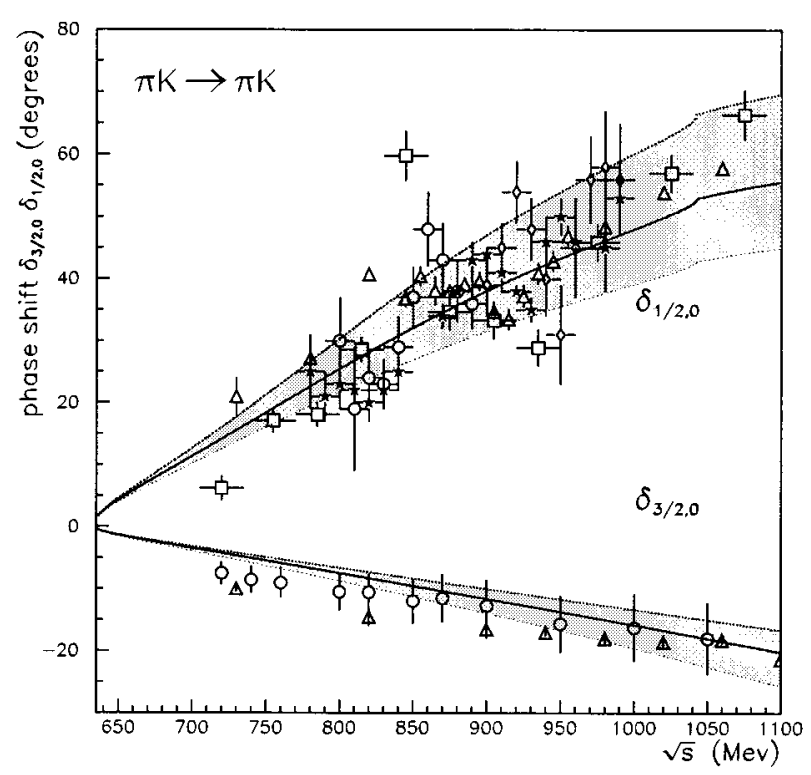

FIG. 4. $\pi K$ elastic scattering phase shifts $\delta_{I J}$ obtained from the IAM fit to the correct $M_{\rho}$ and $M_{K^{*}}$. The shaded areas cover the error bars of the fitted parameters with the constraint $2 \hat{L}_{1}^{r}+\hat{L}_{3}-\hat{L}_{2}^{r}=(-3.11 \pm 0.01) \times 10^{-3}$. The dotted straight line stands at $\delta=90^{\circ}$. The symbols for the experimental data are the same as in Fig. 3. 
TABLE VI. $\pi \pi$ scattering lengths. The one-loop ChPT results are taken from [29]. The experimental data come from [45]. The errors in the IAM fit come only from the uncertainties in the parameters. They do not include other theoretical uncertainties.

\begin{tabular}{cccc}
\hline \hline$a_{J}^{I}$ & ChPT & IAM fit & Experiment \\
\hline$a_{0}^{0}$ & 0.201 & $0.216 \pm 0.008$ & $0.26 \pm 0.05$ \\
$b_{0}^{0}$ & 0.26 & $0.289 \pm 0.025$ & $0.25 \pm 0.03$ \\
$a_{0}^{2}$ & -0.041 & $-0.0417 \pm 0.0014$ & $-0.028 \pm 0.012$ \\
$b_{0}^{2}$ & -0.070 & $-0.075 \pm 0.003$ & $-0.082 \pm 0.008$ \\
$a_{1}^{1}$ & $3.6 \times 10^{-2}$ & $(3.744 \pm 0.002) \times 10^{-2}$ & $(3.8 \pm 0.2) \times 10^{-2}$ \\
$b_{1}^{1}$ & $0.43 \times 10^{-2}$ & $(0.515 \pm 0.001) \times 10^{-2}$ & \\
$a_{2}^{0}$ & $20 \times 10^{-4}$ & $(17.1 \pm 3.5) \times 10^{-4}$ & $(17 \pm 3) \times 10^{-4}$ \\
$a_{2}^{2}$ & $3.5 \times 10^{-4}$ & $(2.8 \pm 1.5) \times 10^{-4}$ & $(1.3 \pm 3.1) \times 10^{-4}$ \\
\hline \hline
\end{tabular}

whole approach should be modified following the comments that we made in previous sections.

In Fig. 4 we show the results of the SU(3) IAM fit to the resonance masses, in terms of elastic scattering phase shifts, which we think deserve some comments.

First, notice that we are not showing the curves for $\pi \pi$ scattering because they are exactly those in Fig. 2 . The differences only appear above the two kaon threshold, since in the $\mathrm{SU}(3)$ formulas we are also considering internal loops of kaons and $\eta$ 's.

In the $\pi K \rightarrow \pi K$ case we can extend the graphs up to $1100 \mathrm{MeV}$, or even more. The reason is that the first two body inelastic threshold is $K \eta$ production at $1040 \mathrm{MeV}$ and, in contrast with the $\pi \pi$ case, there is no nearby resonance. Indeed, the next resonant state in $\pi K$ elastic scattering is $K_{0}^{*}(1430)$, very high to affect dramatically our results at $1100 \mathrm{MeV}$, but also to be correctly reproduced by the IAM method. Nevertheless, the existence of the $K \eta$ threshold can be noticed in the $I=1 / 2, J=0$ channel, as a small bump in the curves at precisely $1040 \mathrm{MeV}$.

The shaded area in the $K^{*}(892)$ channel is not only due to the averaged mass for $K^{*}(892)$ with $2.5 \mathrm{MeV}$ error, but also to the fact that we have to determine several parameters to get the right mass, in contrast with the $\rho(770)$ case, when we only had to fix one.

We have explicitly checked that our ChPT amplitudes satisfy perturbative unitarity. As it is explained in the Appendix, previous calculations [23,13], including ours, did not respect this condition, although by a very small amount. That is why the values of the best parameters for this fit are slightly different from those of our previous work [13].

\section{Phenomenological parameters}

Once we have a good parametrization of $\pi \pi$ and $\pi K$ elastic amplitudes, we can use it to obtain the values of some relevant phenomenological parameters. First, we can calculate the scattering lengths, which determine the strength of the interactions at low energy. Despite the fact that our IAM fit makes use of high-energy data, we expect that it will reproduce the low-energy behavior since in the low-energy
TABLE VII. $\pi K$ scattering lengths. Note that the ChPT results have been obtained using the corrected formulas in the Appendix. The experimental data come from [22].

\begin{tabular}{lccc}
\hline \hline$a_{J}^{I}$ & ChPT & IAM fit & Experiment \\
\hline$a_{0}^{3 / 2}$ & -0.043 & $-0.049 \pm 0.004$ & $-0.13, \ldots,-0.05$ \\
$b_{0}^{3 / 2}$ & & $-0.026 \pm 0.003$ & \\
$a_{0}^{1 / 2}$ & 0.148 & $0.155 \pm 0.012$ & $0.13, \ldots, 0.24$ \\
$b_{0}^{1 / 2}$ & & $0.087 \pm 0.016$ & \\
$a_{1}^{1 / 2}$ & 0.012 & $0.0146 \pm 0.0012$ & $0.017, \ldots, 0.018$ \\
\hline \hline
\end{tabular}

limit it reduces to the chiral expansion, which at $O\left(p^{4}\right)$ already yields quite good values (see Tables VI and VII). However, as the IAM is nonperturbative, we are also taking into account higher order effects, that will modify the results. Indeed, some of these lengths have already been calculated with the IAM and it yields slightly better results than plain ChPT [14]. We have made again the calculation with our fit, but as far as we have an estimate of the error bars in the $\hat{L}_{i}$ parameters, we will also give the error estimates coming only from the uncertainties in $\hat{L}_{i}$ (mostly dominated by that of $\left.\hat{L}_{2}\right)$.

Before giving the results, it is convenient to recall that the scattering lengths have two different normalizations. Namely,

$$
\operatorname{Re} t_{I J}(s)=q^{2 J}\left[a_{J}^{I}+b_{J}^{I} q^{2}+O\left(q^{4}\right)\right]
$$

for $\pi \pi$ scattering, where $q$ is the $\mathrm{cm}$ momentum $q^{2}=s / 4-M_{\pi}^{2}$, and

$$
\operatorname{Re} t_{I J}(s)=\frac{\sqrt{s}}{2} q^{2 J}\left[a_{J}^{I}+b_{J}^{I} q^{2}+O\left(q^{4}\right)\right]
$$

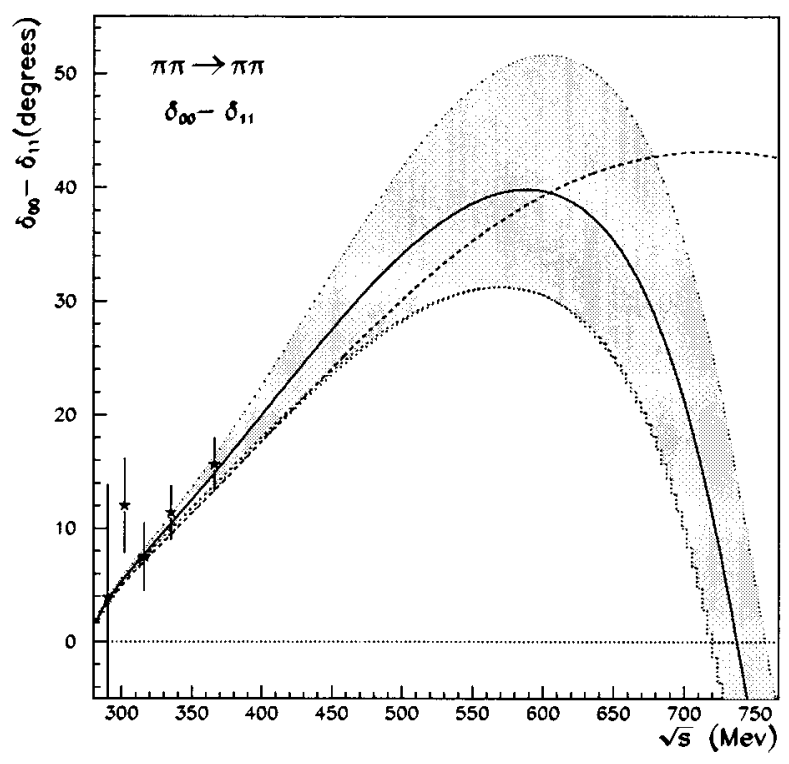

FIG. 5. $\delta_{00}-\delta_{11}$ phase shift difference from the IAM fit (solid line) and plain ChPT (dashed line). The shaded area covers the uncertainty in the $\hat{L}_{i}$ parameters and the data come from [36]. 

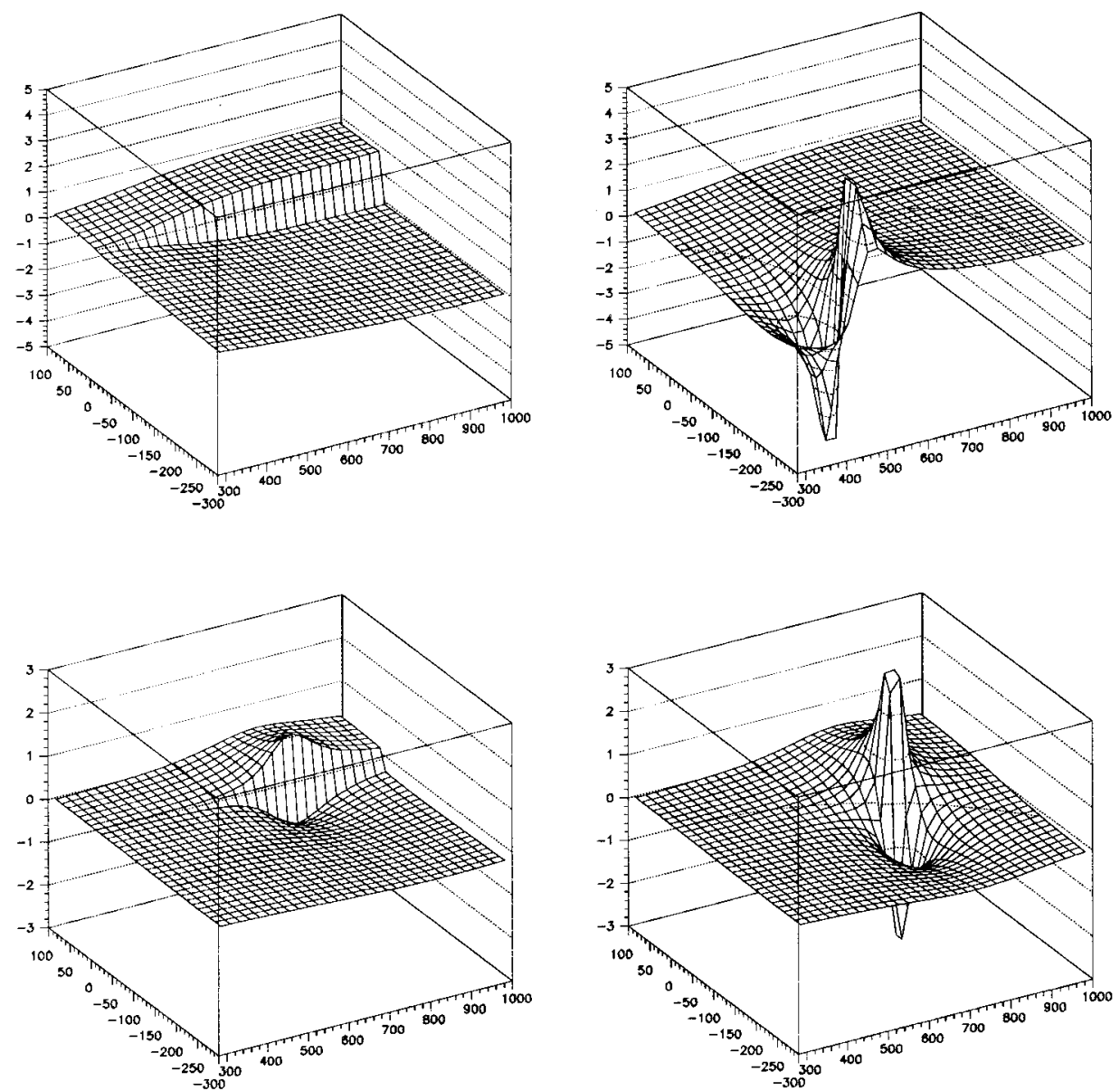

FIG. 6. Imaginary parts of the $\pi \pi \rightarrow \pi \pi$ amplitudes in the complex $s$ plane. The first row is the $(I, J)=(0,0)$ channel, the second is $(1,1)$, and the bottom is $(2,0)$. The left plots correspond to the first Riemann sheet, and those on the right correspond to the second.
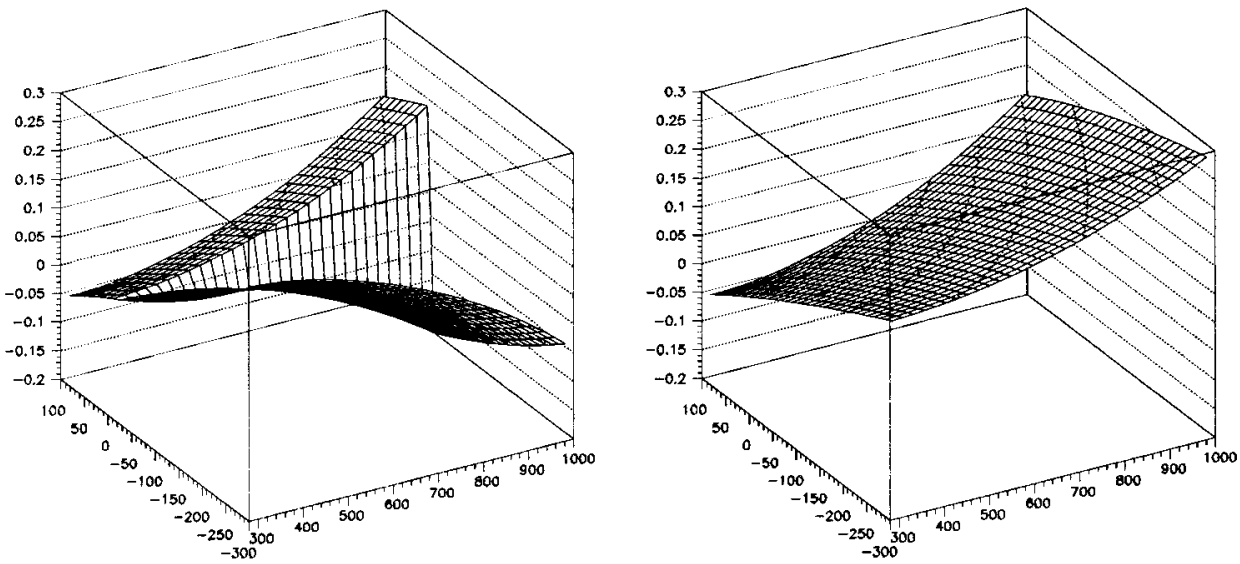

for $\pi K$ scattering, where now $q^{2}=\left[s-\left(M_{K}+M_{\pi}\right)^{2}\right][s$ $\left.-\left(M_{K}-\mathrm{M} \pi\right) 2\right] / 4 \mathrm{~s}$.

The predictions of our fit for the $\pi \pi$ and $\pi K$ scattering lengths are given in Tables VI and VII (in $M_{\pi}$ units). Notice that all the values are compatible with the experimental data, and in general they only differ very slightly from the $O\left(p^{4}\right)$ ChPT results, usually in the right direction toward the central value. However, the experimental error bars are still too big to arrive at any conclusion. Also, the error bars in the IAM have to be interpreted cautiously, since they are obtained only from the uncertainties in the $\hat{L}_{i}$ parameters.

As we have already commented, very recently there has appeared a two-loop calculation of $\pi \pi$ scattering within SU(2) ChPT. It estimates $a_{0}^{0} \sim 0.217$ or 0.215 and $a_{0}^{0}-a_{0}^{2} \sim 0.258$ or 0.256 , which are precisely the values obtained with our IAM fit. This fact gives support to the idea that the IAM somehow takes into account higher order terms even at low energies. 

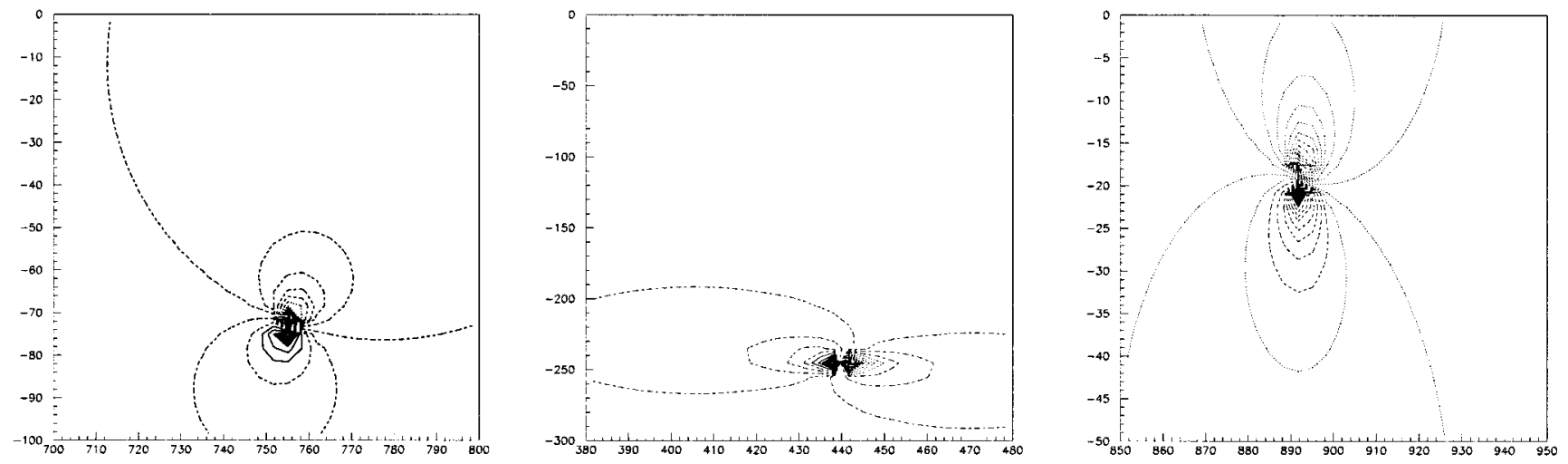

FIG. 7. Contour plots of the second Riemann sheets for different SU(3) ChPT unitarized amplitudes. From left to right they correspond to the $(1,1)$ and $(0,0) \pi \pi$ scattering channels and the $(1 / 2,1) \pi K \rightarrow \pi K$ channel.

Notice that we do not compare our results with the twoloop calculation of the scattering lengths and slopes in [4] because they have used them as an input in a $\chi^{2}$ fit to determine their additional $\alpha$ and $\beta$ parameters. Therefore, their values are almost exactly those of the experimental data. However, as far as there are no data for the $b_{1}^{1} \pi \pi$ slope parameter, their value can be regarded as a prediction. They give $b_{1}^{1}=(0.54 \pm 0.15) \times 10^{-2}$, which is consistent with our result and with $b_{1}^{1}=(0.6 \pm 0.4) \times 10^{-2}$, that was obtained from sum rules in [44].

We have also calculated the phase of the $\epsilon^{\prime}$ parameter, which measures direct $C P$ violation in $K \rightarrow \pi \pi$ decays [46]. It is related to the $s$-wave phase shifts as

$$
\phi\left(\epsilon^{\prime}\right)=90^{\circ}-\left(\delta_{0}^{0}-\delta_{0}^{2}\right)_{s=M_{K^{0}}^{2}} .
$$

Our result is

$$
\phi\left(\epsilon^{\prime}\right)=\left(42_{+5}^{-7}\right)^{\circ},
$$

very close to $\phi\left(\epsilon^{\prime}\right)=(45 \pm 6)^{\circ}$ which is obtained in plain ChPT [47]. In contrast with the case of the scattering lengths, the value of this angle is not used as an input in [4] and is, therefore, a prediction of their best fit. The value they quote is $\phi\left(\epsilon^{\prime}\right)=(43.5 \pm 2 \pm 6)^{\circ}$.

Finally, in Fig. 5 we show the phase difference $\delta_{00}-\delta_{11}$, compared with the available experimental data [36]. The difference between the IAM and plain ChPT at high energies is due to the presence of the $\rho$ resonance. Nevertheless, there are also some differences at low energies, since the dispersive approach is somehow taking into account higher order contributions.

\section{THE IAM IN THE COMPLEX $s$ PLANE}

The main objection to unitarization procedures is the apparent arbitrariness in their predictions, which may differ from one another. In most cases, these methods are nothing but a small modification of the amplitudes so that they can satisfy the unitarity constraint in Eq. (4), while keeping at the same time the good low-energy behavior. But that constraint is not enough to determine the amplitude completely. Thus, there are as many unitarization techniques as algebraic tricks to implement such a constraint exactly or to get a better approximation.
However, we have already seen in Sec. III A that, below any other inelastic threshold, the inverse amplitude method can be derived directly from the analytic structure of the general two body elastic scattering amplitude. Our purpose in this section is to show that, apart from satisfying elastic unitarity, it provides the correct analytic structure required from relativistic quantum field theory. Such a structure is not trivial at all and cannot be reproduced by other unitarization procedures. Both the left and right unitarity cuts are already present in plain ChPT; therefore, we will mainly focus on the poles in the second Riemann sheet.

In the previous section we used the most naive criteria to identify resonances, i.e., that the phase crosses the $\delta=90^{\circ}$ value. However, that is only true for the simplest cases. The rigorous characterization of resonances is made in terms of poles in the second Riemann sheet of the amplitudes in the $s$-complex plane. Indeed, when a resonance is produced by just one of these poles, both its mass and width are related to the pole position by

$$
{\sqrt{s_{\text {pole }}}} \simeq M_{R}+i \frac{\Gamma_{R}}{2},
$$

provided the width is small enough.

In this work we have extended to the $s$-complex plane both the $\pi \pi$ and the $\pi K$ elastic scattering IAM amplitudes obtained in the previous section. Notice that the cuts in ChPT come from logarithmic functions, so that we have infinite sheets in the complex plane. However, only two of them correspond to the first and second Riemann sheets. Once we have identified these sheets we can check whether the resonances that we found in previous sections are produced by a pole in the second Riemann sheet and thus whether they have a real basis.

We will first analyze the $\pi \pi \rightarrow \pi \pi$ process. In Fig. 6 we represent the imaginary part of the amplitude in the complex $s$ plane for the three channels $(I, J)=(0,0),(1,1)$, and $(2,0)$. Notice that when we say complex $s$ plane, we mean that we have parametrized $s$ as $s=(E+i C)^{2}$, where $E$ is the c.m. energy and is represented in the real axis whereas $C$ provides the complex part. On the left column we have displayed the results in the first Riemann sheet, whereas in the right column we have continued through the cut to the lower half of the second Riemann sheet. In all cases the existence of a cut 

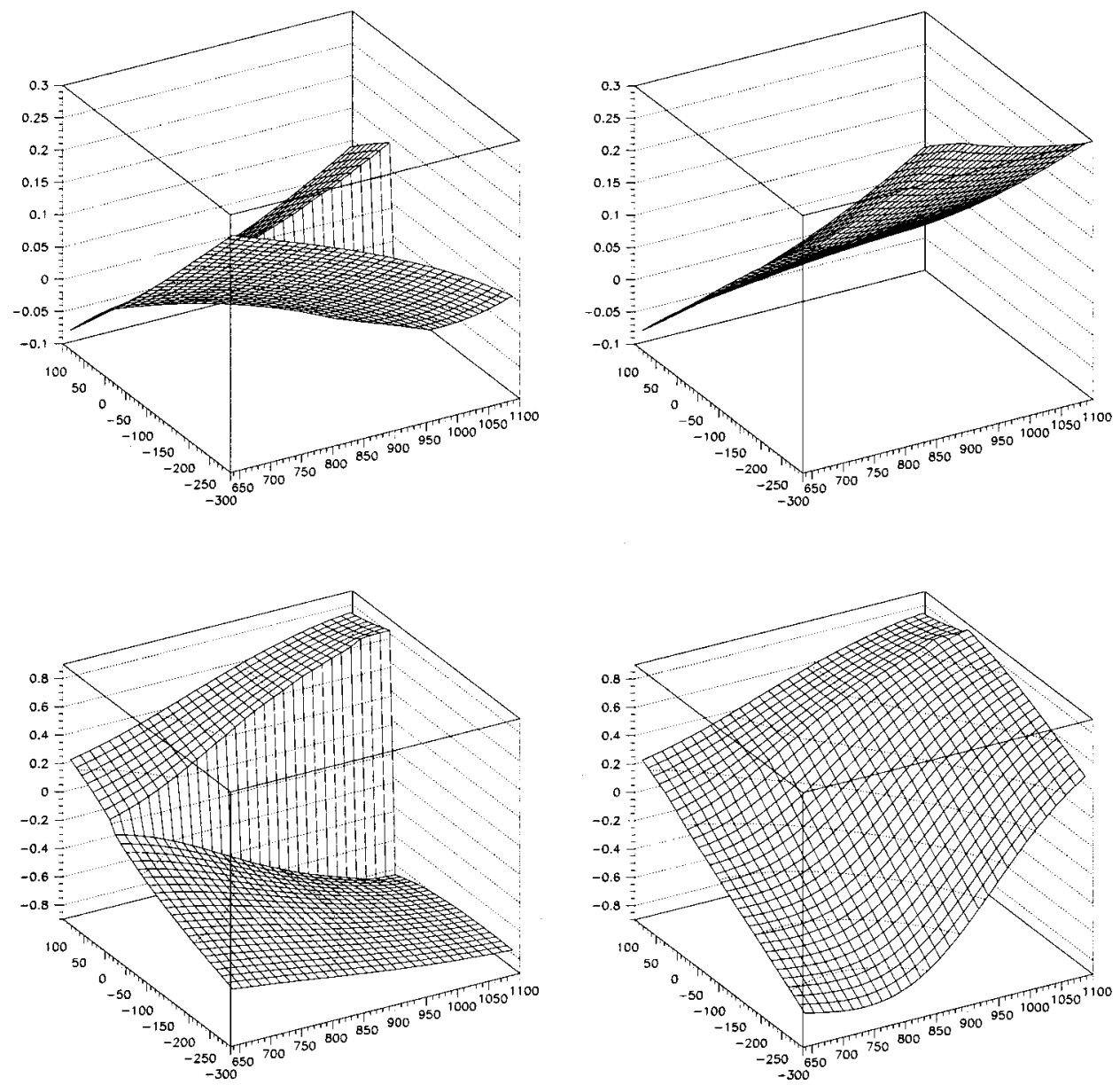

FIG. 8. Imaginary parts of the $\pi K \rightarrow \pi K$ IAM amplitudes in the complex $s$ plane. The first row is the $(I, J)=(3 / 2,0)$ channel, the second is $(1 / 2,0)$, and the bottom is $(1 / 2,1)$. Again, the left plots correspond to the first Riemann sheet, and those on the right correspond to the second.
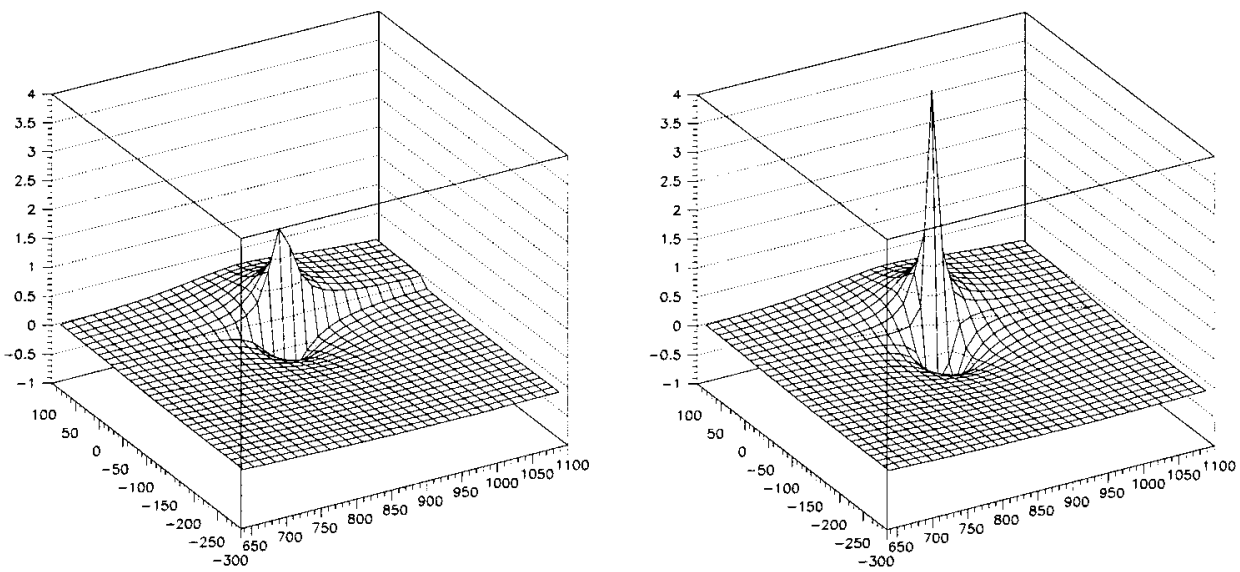

on the real axis on the first Riemann sheet can be clearly noticed. As we had commented before, a right cut is not anything completely new, since it is already present in oneloop ChPT, although in that case, the values that the amplitudes take on it are different. In contrast, the most striking new feature in the IAM amplitudes is the appearance of poles in the second Riemann sheet and how they determine the amplitude shape for the physical values of $s$.

Indeed, we have found two poles with $\operatorname{Im} s<0$ in the second Riemann sheet, one in the $(0,0)$ partial wave and another one in $(1,1)$. Let us start with the second, which clearly corresponds to the $\rho$ resonance. The position of this pole can be obtained from the contour plots in Fig. 7, and it is found at around $E_{R} \sim 760-i 75$. Using Eq. (36) we see that it is in a good agreement with the $\rho(770)$ mass and width parameters given in Table I. Therefore, we can conclude that this pole is completely consistent with the $\rho(770)$ resonance.

The other pole that can be seen in $\pi \pi \rightarrow \pi \pi$ is on the $(0,0)$ channel. Using the parameters of the best SU(3) IAM fit of the previous section, we find that it is located at $E_{R} \sim 440-i 245$. It is not responsible for the appearance of any resonance, since it is very far away from the real axis. However, from purely phenomenological fits to pion scattering data, the existence of such a pole around $E_{R} \sim 408$ $-i 342 \mathrm{MeV}$ had already been pointed out [27]. This pole is responsible for the strong interaction in that it dominates at 
low energy the two pion $(0,0)$ channel. We can now see that even in the channel where there is not an apparent improvement, the IAM yields the correct analytic structure.

Much as it happened in previous sections, the method is not able to reproduce the $f_{0}(980)$ resonance. As we already commented, the interpretation in terms of poles of this resonance is still controversial. Following the same steps as before, we have also identified the four Riemann sheets that now appear due to the superposition of two cuts. Indeed, we have even implemented the IAM derived with the inelastic unitarity condition in Sec. III B 4. We have not found any pole that could hint at the existence of such a resonance.

As we have already explained, we should not expect to find anything since the approach is not able to reproduce properly either the two kaon unitarity cut or, consequently, its associated sheet structure.

Let us now address the $\pi K$ elastic scattering case. Again, in Fig. 8 we have displayed the imaginary part of the amplitudes for the $(3 / 2,0),(1 / 2,0)$, and $(1 / 2,1)$. The pictures on the left represent the first Riemann sheet and those on the right, the second. Once more, the existence of a unitarity cut is clear, but there is also the appearance of a pole in the appropriate channel. In particular, using the third contour plot of Fig. 7, we have found a pole in $E_{R} \sim 890-i 20 \mathrm{MeV}$, which, using Eq. (36), yields again the mass and width for the $K^{*}(892)$ resonance that we gave in Table V.

\section{CONCLUSIONS}

In this work we have shown how the IAM provides a consistent technique to accommodate resonances. Indeed, based on its derivation from dispersion theory, we have made a systematic analysis of its applicability, which is mainly limited by the existence of two body inelastic thresholds and by the fact that the tree-level approximation vanishes in some channels.

We have found that it is possible to predict the most relevant features of strong elastic scattering, once the chiral parameters are determined from low-energy data. Quantitatively, the errors are hard to estimate, but we have found that in all cases the mass of the predicted resonances falls within approximately $15 \%$ of their actual values. We think this fact gives a sound basis for its application in order to obtain at least a qualitative description of resonances in the strongly interacting symmetry-breaking sector.

Moreover, once we force the IAM results to fit the actual resonance mass values, we get a remarkably good fit which is able to reproduce the experimental data up to the next relevant two body inelastic threshold. Following that procedure, we have given the unitarized $\mathrm{SU}(2) \mathrm{ChPT}$ fit to $\pi \pi \rightarrow \pi \pi$ as well as that of $\mathrm{SU}(3)$ to $\pi \pi$ and $\pi K$ elastic scattering. For the first time we have estimated the values of the unitarized chiral parameters together with their error bars. These values do not lie very far from those obtained without the IAM and, therefore, do not spoil the low-energy expansion, as can be noticed from the scattering lengths that we have given.

With this fit, we have calculated several low-energy phenomenological parameters, such as the scattering lengths. Our values differ from those obtained at $O\left(p^{4}\right)$ due to the unitarization. However, we expect that they include other corrections due to unitarity and resonant effects.

We consider that it would not make any sense to try to reduce the error bars in the unitarized parameters within this approach. One has to keep in mind that we have neglected higher order ChPT corrections, isospin-breaking contributions, and that we have used high-energy data which is very sensitive in such effects. It is quite likely that, in order to obtain results consistent to a higher degree of accuracy, the IAM in the simple version that has been used here will not be enough.

Finally, we have also shown how the IAM yields the proper analytic structure in the complex $s$ plane, in contrast with other unitarization techniques. Indeed, we have found that the apparent resonant behavior that is observed on phase shifts is produced by the corresponding poles in the second Riemann sheet, meeting the strict requirements imposed by general relativistic quantum field theory.

Therefore, we think that the IAM and unitarization by means of dispersion theory is the most natural and economic way to extend the applicability of chiral Lagrangians. We have seen however, that its main limitations come from the existence of two body inelastic thresholds. Nevertheless, work is still in progress on the subject, the IAM has been recently applied to other processes, and higher order ChPT calculations will be soon available. Since some other physically relevant features do not lie very far from the present applicability limits, it seems very likely that they can be reproduced in the near future.

\section{ACKNOWLEDGMENTS}

J.R.P would like to thank the Theory Group at Berkeley for their kind hospitality and the Jaime del Amo Foundation for financial support. He also wishes to thank M. S. Chanowitz for calling his attention to the $f_{0}$ problem. We have also profitted from discussions with U. G. Meissner on the $\pi K$ elastic scattering amplitudes and M. R. Pennington on the Adler zero problem and the left cut contribution. We thank D. Toublan too, for pointing an error in our calculations as well as C. Carone for reading the manuscript. This work has been partially supported by the Ministerio de Educación y Ciencia (Spain) (CICYT AEN93-0776). Partial support by U.S. DOE under Contract No. DE-AC03-76SF00098 is gratefully acknowledged.

\section{APPENDIX: ELASTIC SCATTERING AMPLITUDES IN SU(3) ChPT AND UNITARITY}

The first calculation of elastic $\pi K$ scattering was performed in [22]. These amplitudes were given in terms of physical as well as lowest order masses and decay constants, which are usually denoted by $M_{P}, F_{P}$ and $M_{P}^{0}, F^{0}$, respectively ( $P$ being either $\pi, K$, or $\eta$ ). Of course, the only measurable parameters are the first, and when comparing with experimental observations, one has to eliminate those from lowest order in terms of the physical constants.

Indeed, it is possible to find [3] the relation between $M_{\pi}^{0}$ and $M_{\pi}$ as well as that between $M_{K}^{0}$ and $M_{K}$. Unfortunately, at lowest order there is only one $F^{0}$, which is related both to $F_{\pi}$ and $F_{K}$. Hence, whenever one finds $F^{0}$ in an expression 
there are two choices: relate it either to $F_{\pi}$ or to $F_{K}$. The difference between the two choices will be one order higher in the chiral expansion. For instance, if one has an $O\left(p^{2}\right)$ expression with $F_{0}^{2}$, in principle, one can substitute it by $F_{\pi}^{2}, F_{K}^{2}$, or $F_{\pi} \cdot F_{K}$. All these choices are equally acceptable. When one is working only with pions, the natural choice is the one that leaves all the expressions in terms of $F_{\pi}$. When one is dealing both with pions and kaons, it is not so obvious. However, once one choice is made for the $O\left(p^{2}\right)$ term, we have to keep it for the $O\left(p^{4}\right)$ contribution, otherwise one would violate perturbative unitarity, Eq. (4).

Unfortunately, in the amplitude in the literature [23], which is the one we had also followed in our previous work
[13], the choice for the $O\left(p^{2}\right)$ term is different from that of the $O\left(p^{4}\right)$ contribution that yields the imaginary part. Indeed, the $O\left(p^{2}\right)$ term is written just in terms of $F_{\pi}$ whereas $T^{U}$ is written in terms of $F_{\pi}$ and $F_{K}$. As a consequence, there is a $F_{K}^{2} / F_{\pi}^{2}$ factor of difference between $\operatorname{Im} t^{(1)}$ and $\sigma_{\pi K}\left|t^{(0)}\right|^{2}$. Numerically, that amounts to a $(1.22)^{2} \simeq 1.5$ factor.

Thus, we have rederived from the original work [22] the amplitudes in terms of physical quantities (also correcting some small errors), so that they satisfy perturbative unitarity. We have chosen to write the formulas symmetrically with respect to $F_{\pi}$ and $F_{K}$. But the other choices are equally acceptable. The result is

$$
\begin{aligned}
& T^{3 / 2}(s, t, u)=\frac{M_{\pi}^{2}+M_{K}^{2}-s}{2 F_{\pi} F_{K}}+T_{4}^{T}(s, t, u)+T_{4}^{P}(s, t, u)+T_{4}^{U}(s, t, u)+O\left(s^{3}\right), \\
& T_{4}^{T}(s, t, u)=\frac{1}{16 F_{\pi} F_{K}}\left(M_{\pi}^{2}-M_{K}^{2}\right)\left(3 \mu_{\pi}-2 \mu_{K}+\mu_{\eta}\right), \\
& T_{4}^{P}(s, t, u)=\frac{2}{F_{\pi}^{2} F_{K}^{2}}\left\{4 L_{1}^{r}\left(t-2 M_{\pi}^{2}\right)\left(t-2 M_{K}^{2}\right)+2 L_{2}^{r}\left[\left(s-M_{\pi}^{2}-M_{K}^{2}\right)^{2}+\left(u-M_{\pi}^{2}-M_{K}^{2}\right)^{2}\right]\right. \\
& +L_{3}^{r}\left[\left(u-M_{\pi}^{2}-M_{K}^{2}\right)^{2}+\left(t-2 M_{\pi}^{2}\right)\left(t-2 M_{K}^{2}\right)\right]+4 L_{4}^{r}\left[t\left(M_{\pi}^{2}+M_{K}^{2}\right)-4 M_{\pi}^{2} M_{K}^{2}\right] \\
& \left.+2 L_{5}^{r} M_{\pi}^{2}\left(M_{\pi}^{2}-M_{K}^{2}-s\right)+8\left(2 L_{6}^{r}+L_{8}^{r}\right) M_{\pi}^{2} M_{K}^{2}\right\}, \\
& T_{4}^{U}(s, t, u)=\frac{1}{4 F_{\pi}^{2} F_{K}^{2}}\left\{\frac{3}{2}\left[(s-t)\left\{L_{\pi K}(u)+L_{K \eta}(u)-u\left[M_{\pi K}^{r}(u)+M_{K \eta}^{r}(u)\right]\right\}+\left(M_{K}^{2}-M_{\pi}^{2}\right)^{2}\left[M_{\pi K}^{r}(u)+M_{K \eta}^{r}(u)\right]\right]+t(u-s)\right. \\
& \times\left[2 M_{\pi \pi}^{r}(t)+M_{K K}^{r}(t)\right]+\frac{1}{2}\left(M_{K}^{2}-M_{\pi}^{2}\right)\left[K_{\pi K}(u)\left(5 u-2 M_{K}^{2}-2 M_{\pi}^{2}\right)+K_{K \eta}(u)\left(3 u-2 M_{K}^{2}-2 M_{\pi}^{2}\right)\right]+\frac{1}{8} J_{\pi K}^{r}(u) \\
& \times\left[11 u^{2}-12 u\left(M_{K}^{2}+M_{\pi}^{2}\right)+4\left(M_{K}^{2}+M_{\pi}^{2}\right)^{2}\right]+J_{\pi K}^{r}(s)\left(s-M_{K}^{2}-M_{\pi}^{2}\right)^{2}+\frac{3}{8} J_{K \eta}^{r}(u)\left(u-\frac{3}{2}\left(M_{K}^{2}+M_{\pi}^{2}\right)\right)^{2} \\
& \left.+\frac{1}{2} J_{\pi \pi}^{r}(t) t\left(2 t-M_{\pi}^{2}\right)+\frac{3}{4} J_{K K}^{r}(t) t^{2}+\frac{1}{2} J_{\eta \eta}^{r}(t) M_{\pi}^{2}\left(t-\frac{8}{9} M_{K}^{2}\right)\right\}
\end{aligned}
$$

The functions $M_{P Q}, L_{P Q}, K_{P Q}, J_{P Q}, \mu_{P}$, with $P, Q=\pi, K, \eta$, can be found in [3] although they should be written in terms of physical quantities.

We have verified analytically that this amplitude satisfies the perturbative unitarity constraint. Moreover, we have used that constraint as a check of our programs.

We want to remark again that this way to write the $\pi K$ amplitude is one of several possible choices, since we could have chosen to write everything just in terms of $F_{\pi}$, for example. The important point is to keep the same choice $b o t h$ for the $O\left(p^{2}\right)$ and the $O\left(p^{4}\right)$.

For completeness, we will also give the SU(3) formulas used in this work for $\pi \pi$ scattering, because they have also appeared with some minor errors in the literature:

$$
\begin{aligned}
& A(s, t, u)=\frac{\left(s-M_{\pi}^{2}\right)}{F_{\pi}^{2}}+B(s, t, u)+C(s, t, u)+O\left(s^{3}\right), \\
& B(s, t, u)= \frac{1}{F_{\pi}^{4}}\left\{\frac{M_{\pi}^{4}}{18} J_{\eta \eta}^{r}(s)+\frac{1}{2}\left(s^{2}-M_{\pi}^{4}\right) J_{\pi \pi}^{r}(s)+\frac{1}{8} s^{2} J_{K K}^{r}(s)\right. \\
&\left.+\frac{1}{4}\left(t-2 M_{\pi}^{2}\right)^{2} J_{\pi \pi}^{r}(t)+t(s-u)\left[M_{\pi \pi}^{r}(t)+\frac{1}{2} M_{K K}^{r}(t)\right]+(t \leftrightarrow u)\right\},
\end{aligned}
$$


$C(s, t, u)=\frac{4}{F_{\pi}^{4}}\left\{\left(2 L_{1}^{r}+L_{3}\right)\left(s-2 M_{\pi}^{2}\right)^{2}+L_{2}^{r}\left[\left(t-2 M_{\pi}^{2}\right)^{2}+\left(u-2 M_{\pi}^{2}\right)^{2}\right]+\left(4 L_{4}^{r}+2 L_{5}^{r}\right) M_{\pi}^{2}\left(s-2 M_{\pi}^{2}\right)+\left(8 L_{6}^{r}+4 L_{8}^{r}\right) M_{\pi}^{4}\right\}$

[1] S. Weinberg, Physica A 96, 327 (1979).

[2] J. Gasser and H. Leutwyler, Ann. Phys. (N.Y.) 158, 142 (1984).

[3] J. Gasser and H. Leutwyler, Nucl. Phys. B250, 465 (1985); B250, 517 (1985).

[4] K. Knecht, B. Moussallam, J. Stern, and N. H. Fuchs, Nucl. Phys. B457, 513 (1995).

[5] J. Bijnens, G. Colangelo, G. Ecker, J. Gasser, and M. E. Sainio, Phys. Lett. B 374, 210 (1996).

[6] Chiral Dynamics: Theory and Experiment, Proceedings of the Workshop held at MIT, Cambridge, MA, July, 1994, edited by A. M. Bernstein and B. R. Holstein (Springer, Berlin, 1995).

[7] Tran N. Truong, Phys. Rev. Lett. 61, 2526 (1988); 67, 2260 (1991).

[8] A. Dobado, M. J. Herrero, and T. N. Truong, Phys. Lett. B 235, 134 (1990).

[9] G. Ecker, J. Gasser, A. Pich, and E. de Rafael, Nucl. Phys. B321, 311 (1989); G. Ecker, J. Gasser, H. Leutwyler, A. Pich, and E. de Rafael, Phys. Lett. B 223, 425 (1989); J. F. Donoghue, C. Ramirez, and G. Valencia, Phys. Rev. D 39, 1947 (1989); V. Bernard, N. Kaiser, and U. G. Meissner, Nucl. Phys. B364, 283 (1991).

[10] M. Harada, F. Sannino, and J. Schechter, Phys. Rev. D 54, 1991 (1996).

[11] S. N. Gupta, Quantum Electrodynamics (Gordon and Breach, New York, 1981), p. 191.

[12] C. J. C. Im, Phys. Lett. B 281, 357 (1992); A. Dobado and J. R. Peláez, ibid. 286, 136 (1992): A. Dobado and J. Morales, ibid. 365, 264 (1996); Phys. Rev. D 52, 2878 (1995).

[13] A. Dobado and J. R. Peláez, Phys. Rev. D 47, 4883 (1993).

[14] T. Hannah, Phys. Rev. D 51, 103 (1995); 52, 4971 (1995).

[15] A. Dobado and J. R. Peláez, Z. Phys. C 57, 501 (1993).

[16] Particle Data Group (PDG), L. Montanet et al., Phys. Rev. D 50, 1173 (1994), and 1995 off-year partial update for the 1996 edition available on the PDG WWW pages (URL:http:// pdg.lbl.gov).

[17] J. M. Cornwall, D. N. Levin, and G. Tiktopoulos, Phys. Rev. D 10, 1145 (1974); B. W. Lee, C. Quigg, and H. Thacker, ibid. 16, 1519 (1977); M. Veltman, Acta Phys. Pol. B 8, 475 (1977); M. S. Chanowitz and M. K. Gaillard, Nucl. Phys. B261, 379 (1985).

[18] T. Appelquist and C. Bernard, Phys. Rev. D 22, 200 (1980); A. C. Longhitano, Nucl. Phys. B188, 118 (1981).

[19] A. Dobado and M. J. Herrero, Phys. Lett. B 228, 495 (1989); 233, 505 (1989); J. Donoghue and C. Ramirez ibid. 234, 361 (1990); A. Dobado, M. J. Herrero, and T. N. Truong, ibid. 235, 129 (1989); A. Dobado, M. J. Herrero, and J. Terrón, Z. Phys. C 50, 205 (1991); 50, 465 (1991); S. Dawson and G. Valencia, Nucl. Phys. B352, 27 (1991); A. Dobado, D. Espriu, and M. J.
Herrero, Phys. Lett. B 255, 405 (1991); D. Espriu and M. J. Herrero, Nucl. Phys. B373, 117 (1992).

[20] A. Dobado, M. J. Herrero, J. R. Peláez, E. Ruiz Morales, and M. T. Urdiales, Phys. Lett. B 352, 400 (1995); CMS Technical Proposal, CERN/LHC94-38, LHCC/P1, 1994.

[21] A. Dobado and J. R. Peláez, Nucl. Phys. B425, 110 (1994); Phys. Lett. B 329, 469 (1994); 335, 554 (1994); H. J. He, Y. P. Kuang, and X. Li, ibid. 329, 278 (1994).

[22] V. Bernard, N. Kaiser, and U. G. Meissner, Phys. Rev. D 43, 2757 (1991).

[23] V. Bernard, N. Kaiser, and U. G. Meissner, Nucl. Phys. B357, 129 (1991).

[24] J. Gasser and U. G. Meissner, Nucl. Phys. B357, 90 (1991); Phys. Lett. B 258, 219 (1991).

[25] The Second DAФNE Physics Handbook, edited by L. Maiani, G. Pancheri, and N. Paver (INFN, Frascati, 1995).

[26] K. L. Au, D. Morgan, and M. R. Pennington, Phys. Rev. D 35, 1633 (1987); D. Morgan and M. R. Pennington, ibid. 48, 1185 (1993); G. Jansen et al., ibid. 52, 2690 (1995): N. Tornqvist and M. Roos, Phys. Rev. Lett. 76, 1575 (1996).

[27] B. S. Zou and D. V. Bugg, Phys. Rev. D 48, 3948 (1993).

[28] H. Lehmann, Phys. Lett. 41B, 529 (1972).

[29] C. Riggenbach, J. F. Donogue, J. Gasser, and B. Holstein, Phys. Rev. D 43, 127 (1991).

[30] J. Bijnens, G. Colangelo, and J. Gasser, Nucl. Phys. B427, 427 (1994).

[31] S. D. Protopopescu et al., Phys. Rev. D 7, 1279 (1973).

[32] G. Grayer et al., Nucl. Phys. B75, 189 (1974).

[33] M. J. Losty et al., Nucl. Phys. B69, 185 (1974).

[34] P. Estabrooks and A. D. Martin, Nucl. Phys. B79, 301 (1974).

[35] V. Srinivasan et al., Phys. Rev. D 12, 681 (1975).

[36] L. Rosselet et al., Phys. Rev. D 15, 574 (1977).

[37] W. Hoogland et al., Nucl. Phys. B126, 109 (1977).

[38] R. Mercer et al., Nucl. Phys. B32, 381 (1971).

[39] H. H. Bingham et al., Nucl. Phys. B41, 1 (1972).

[40] D. Linglin et al., Nucl. Phys. B57, 64 (1973).

[41] M. J. Matison et al., Phys. Rev. D 9, 1872 (1974).

[42] S. L. Baker et al., Nucl. Phys. B99, 211 (1975).

[43] P. Estabrooks et al., Nucl. Phys. B133, 490 (1978).

[44] B. Ananthanarayan, D. Toublan, and G. Wanders, Phys. Rev. D 53, 2362 (1996).

[45] J. L. Basdevant, C. D. Froggat, and J. L. Petersen, Nucl. Phys. B72, 413 (1974); J. L. Basdevant, P. Chapelle, C. López, and M. Sigelle, ibid. B98, 285 (1975); C. D. Froggat and J. L. Petersen, ibid. B129, 89 (1977); J. L. Petersen, The $\pi \pi$ interaction, CERN Yellow Report No. 77-04 (1977).

[46] T. T. Wu and C. N. Yang, Phys. Rev. Lett. 13, 380 (1964).

[47] J. Gasser and U.-G. Meissner, Phys. Lett. B 258, 129 (1991).

[48] M. Boglione and M. R. Pennington, Report No. DTP-96/60, hep-ph/9607266. 